OPEN ACCESS

Edited by:

Zhi Wang,

Innovation Academy for Precision

Measurement Science and

Technology (CAS), China

Reviewed by:

Khitam Muhsen,

Tel Aviv University, Israel

Naga Raju Maddela,

Technical University of Manabi,

Ecuador

*Correspondence:

Weiqian Tian

weiqian@kth.se

Lanqing Ma

lqma@bua.edu.cn

${ }^{+}$These authors have contributed equally to this work

Specialty section:

This article was submitted to

Toxicology, Pollution and the

Environment

a section of the journal

Frontiers in Environmental Science

Received: 08 April 2021 Accepted: 03 June 2021 Published: 18 June 2021

Citation:

Liu C, Tan L, Zhang L, Tian W and Ma L (2021) A Review of the Distribution of Antibiotics in Water in Different Regions of China and Current Antibiotic

Degradation Pathways.

Front. Environ. Sci. 9:692298.

doi: 10.3389/fenvs.2021.692298

\section{A Review of the Distribution of Antibiotics in Water in Different Regions of China and Current Antibiotic Degradation Pathways}

\author{
Can $\mathrm{Liu}^{1 \dagger}$, Li Tan ${ }^{1 \dagger}$, Liming Zhang $^{2}$, Weiqian Tian ${ }^{2 *}$ and Lanqing $\mathrm{Ma}^{1 *}$ \\ ${ }^{1}$ Key Laboratory for Northern Urban Agriculture of Ministry of Agriculture and Rural Affairs, Beijing University of Agriculture, Beijing, \\ China, ${ }^{2}$ Department of Fibre and Polymer Technology, KTH Royal Institute of Technology, Stockholm, Sweden
}

Antibiotic pollution is becoming an increasingly serious threat in different regions of China. The distribution of antibiotics in water sources varies significantly in time and space, corresponding to the amount of antibiotics used locally. The main source of this contamination in the aquatic environment is wastewater from antibiotic manufacturers, large scale animal farming, and hospitals. In response to the excessive antibiotic contamination in the water environment globally, environmentally friendly alternatives to antibiotics are being developed to reduce their use. Furthermore, researchers have developed various antibiotic treatment techniques for the degradation of antibiotics, such as physical adsorption, chemical oxidation, photodegradation, and biodegradation. Among them, biodegradation is receiving increasing attention because of its low cost, ease of operation, and lack of secondary pollution. Antibiotic degradation by enzymes could become the key strategy of management of antibiotics pollution in the environment in future. This review summarizes research on the distribution of antibiotics in China's aquatic environments and different techniques for the degradation of antibiotics. Special attention is paid to their degradation by various enzymes. The adverse effects of the pollutants and need for more effective monitoring and mitigating pollution are also highlighted.

Keywords: antibiotic contamination, antibiotic resistance, enzyme degradation, water environment, ecosystems

\section{INTRODUCTION}

Since Fleming discovered penicillin in 1929, hundreds of other antibiotics have been synthesized, which are being increasingly used to treat infections in humans and animals. Inexpensive and effective antibiotics have become the preferred antibacterial drugs used by pharmaceutical and farming industries to inhibit the growth of bacteria and eliminate pathogens. In the aquaculture industry, antibiotics are used extensively as drugs to prevent bacterial infections and parasitic diseases (Bitchava and Nengas, 2010). Only a small portion of the antibiotics in aquatic products are actually absorbed, with most being discharged into the environment, resulting in antibiotic residues in aquaculture areas in discharged wastewaters and accumulated in the surrounding sediments through adsorption (Kumar et al., 2005; Conkle et al., 2010; Rico et al., 2013). In livestock farming, antibiotics are important for the prevention of infectious diseases and their treatment as well as for promoting the growth of livestock (Yin et al., 2016). Antibiotics applied to livestock and poultry are 
not fully absorbed, with most being excreted into the environment through animal feces or urine (Briones et al., 2016). Residual antibiotics enter rivers and lakes through wastewater and accumulate in soil, where they are taken up by plants and animals (as illustrated in Figure 1).

As the world's most populous country and the largest consumer of antibiotics, China's antibiotic stewardship is facing significant challenges (Shao et al., 2020). Some of the antibiotics detected have been banned in clinical practice and may seriously impair human immunity (Zhou et al., 2021). In addition, exposure to veterinary antibiotics is associated with childhood obesity (Scott et al., 2016; Park et al., 2020) and liver injury (Mosedale et al., 2014), and the resulting genetic contamination of resistance poses serious threats to human health. China is vast territory with an equally diverse industrial layout. In addition, the levels of economic development vary greatly across the country, and thus the range of antibiotic concentrations in the environment is also broad across different regions. Here, first, we review the distribution of antibiotics in aquatic environments in China and the types of antibiotics, and report the distribution characteristics of antibiotics in China. Secondly, we analyze the potential impacts of antibiotics on the ecological environment in China. Lastly, we review progress in technologies for the degradation and removal of antibiotics in China and abroad, in addition to exploring the underlying principles, as well as their merits and shortcomings. This review provides a basis for risk estimation of antibiotics in ecological systems, an overview of the distribution of antibiotics in the aquatic environment in China, and the current approaches and methods used to eliminate antibiotics from ecological systems.

\section{STATUS OF ANTIBIOTIC CONTAMINATION IN AQUATIC ENVIRONMENTS IN CHINA}

\section{Types of Antibiotics in Aquatic Environments in China}

The major antibiotics in aquatic environments in China are divided according to their chemical structures and include macrolides, tetracyclines, fluoroquinolones, sulfonamides, and chloramphenicol (Liu et al., 2012). Their structural formulas are listed in Table $\mathbf{1 .}$

\section{Antibiotics in Surface Water}

In different regions of China, the spatial and temporal distribution of antibiotics in water sources varies significantly, and this difference is closely related to the local industrial structure, the mode of antibiotic disposal in the pharmaceutical industry, and the mode of antibiotic use in the livestock industry. As shown in Table 2 by the results of a statistical analysis of data from the last five years, the distribution of antibiotics have been found to vary in different areas, and their contamination level also varies from region to region (Zhang et al., 2019). The antibiotic pollution is mainly concentrated in the Yangtze River Basin, and the Bohai Bay and Pearl River Delta, and Xinjiang regions. In the western region, the medical safety standards are relatively poor, and

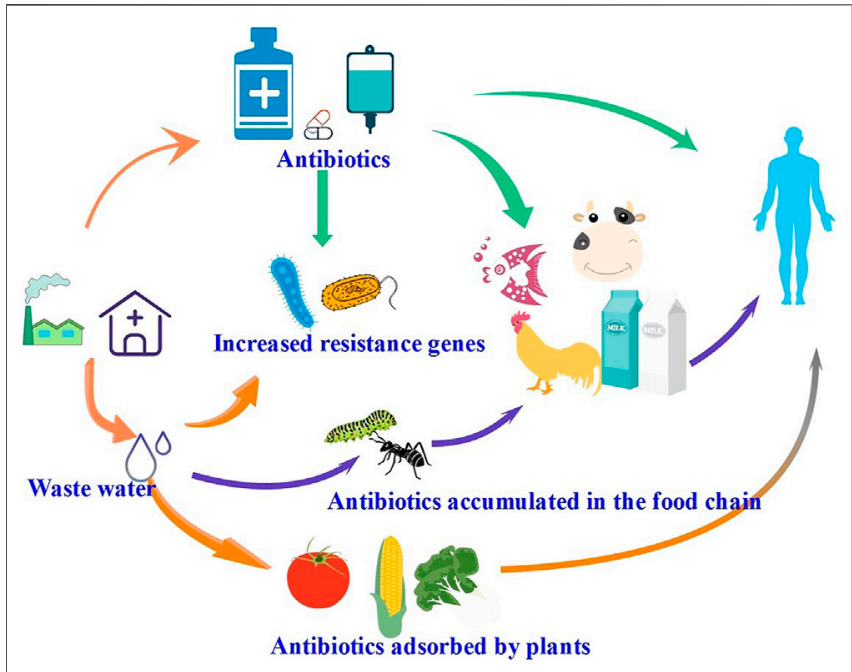

FIGURE 1 | Circulation of antibiotics in the environment.

chloramphenicol, a highly effective antibiotic with relatively greater side effects, is a major problem. As a result of its continued use, the detection rate of chloramphenicol in the middle and upper reaches of the Yangtze River is significantly higher than that in the eastern region (Wang, 2020a). The pharmaceutical industry is concentrated in the economically developed eastern region, leading to the discharge of a large amount of antibiotic effluent and thus a high concentration of antibiotic pollution in this region (Bao et al., 2021). In Xinjiang, coastal areas, and other regions, the development of the livestock and aquaculture industries, which use antibiotics, has resulted in significantly higher levels of sulfonamides and tetracycline antibiotics in the environment. (Table 3). Quinolone antibiotics are widely used as broad-spectrum anti-infective drugs in medical treatment, and their presence has been detected in most local drinking water sources (Table 4). Generally, Chinese rivers and lakes have high concentrations of antibiotics. Sulfonamide and quinolone are the main pollutants in the surface waters of Chinese lakes (Liu et al., 2018).

Antibiotics in the aquatic environment may be influenced by photolysis, temperature, $\mathrm{pH}$, dilution factors, bacterial populations, and hydraulic residence time, leading to inconsistencies in their concentrations (Kummerer, 2009; Kümmerer, 2009; Zhang et al., 2014; Tang et al., 2015). Based on the results of previous research (Yoshizaki and Tomida, 2000; Loftin et al., 2008; Ben et al., 2013), the concentrations and compositions of the main types of antibiotics in the abundant water period and dry water periods are shown in Figure 2, which show that the concentrations of antibiotics in aquatic environments vary seasonally, with detectable frequencies and average concentrations being higher in winter (dry water period) than in summer (dry water period). Industrial structure, medical level, and climate of different regions have an effect on the distribution of antibiotics in local water bodies, especially pharmaceutical and farming industries have a marked effect on antibiotic discharge. Therefore, strengthening guidance and 
TABLE 1 | Major classes of antibiotics in aquatic environments in China.

\begin{tabular}{|c|c|c|c|c|}
\hline Type & Name & Abbreviation & Structure & CAS No. \\
\hline \multirow[t]{2}{*}{ Macrolides } & Roxithromycin & ROX & & 80214-83-1 \\
\hline & Erythromycin & ERM & & $114-07-8$ \\
\hline \multirow[t]{3}{*}{ Tetracyclines } & Tetracycline & $\mathrm{TC}$ & & $60-54-8$ \\
\hline & Oxytetracycline & OTC & & $79-57-2$ \\
\hline & Chlorotetracycli & СTC & & $57-62-5$ \\
\hline & Doxycycline & DOX & & $209-271-1$ \\
\hline \multirow[t]{2}{*}{ Fluoroquinolones } & Ofloxacin & OFX & & 82419-36-1 \\
\hline & Ciprofloxacin & $\mathrm{CIP}$ & & 85721-33-1 \\
\hline
\end{tabular}


TABLE 1 | (Continued) Major classes of antibiotics in aquatic environments in China.

Type

Enoxacin

Enrofloxacin

Sulfonamides

Sulfamethizole

Sulfathiazole

STZ

Sulfamerazine

SMR

Sulfaquinoxaline

SQX

Sulfamonomethoxine

SMM

Sulfapyridine

SPD

Chloramphenicols

Sulfamethoxazole

SMX

Sulfadiazine

Trimethoprim

NOR

SMT

TMP<smiles>CCn1cc(C(=O)O)c(=O)c2cc(F)c(N3CCNCC3)cc21</smiles>

Structure

CAS No.

70458-96-7

74011-58-8<smiles>C=CN(CC)C(C)=NC(C)N1CCNCC1</smiles>

93106-60-6<smiles>CCN1CCN(C(C)C=C2CCCCC2)CC1</smiles>
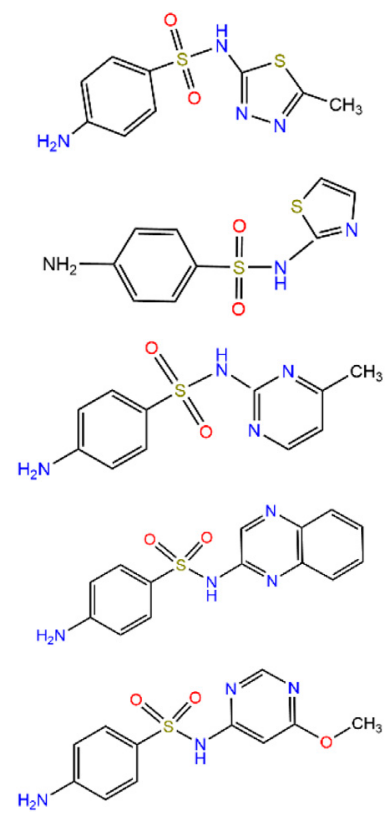<smiles>Nc1ccc(S(=O)(=O)Nc2ccccn2)cc1</smiles><smiles>Cc1cc(NS(=O)(=O)c2ccc(N)cc2)no1</smiles>

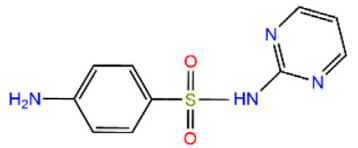

723-46-6

144-82-1

72-14-0

127-79-7

$59-40-5$

144-83-2

$68-35-9$

738-70-5<smiles>COc1cc(Cc2cnc(N)nc2N)cc(OC)c1OC</smiles> 
TABLE 1 | (Continued) Major classes of antibiotics in aquatic environments in China.

Type

Name

Abbreviation

CAP

Chloramphenicol

Thiamphenicol

TAP

Florfenicol<smiles>CS(=O)(=O)c1ccc([C@@H](O)[C@H](CO)NC(=O)C(Cl)Cl)cc1</smiles>

Structure

CAS No.

$56-75-7$

$15318-45-3$

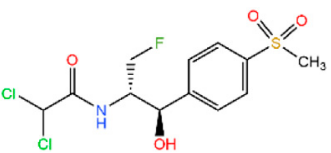

$73231-34-2$

TABLE 2 | Distribution of antibiotics in different waters of China (From 2015 to 2020).

Waters

Regions

Songhua river
Lancang river
Yarlung zangbo river
Huangpu river
Yangtze river (nanjing
section)

Moon lake

Taihu lake basin (yilitaohang section)

Taihu gonghu bay

Tiaoxi (taihu lake basin)

Poyang lake

Chaohu

South lake

Shahu

East lake

Datong lake

Yangtze river basin

(three gorges Section)

Nanming rive

Weihe river (Xi'an

section)

Pearl river

Star lake

Caohai karst plateau

wetland

Huaihe (shihe district)

Xiaoqinghe (jinan

section)

Ebinur lake

Bosten lake

Ebinur lake
Harbin

Lincang

Lasa

Shanghai

Nanjing

Ningbo

Changzhou-wuxi

Wuxi

Huzhou

Nanchang

Hefei

Wuhan

Wuhan

Wuhan

Yiyang

Chongqing

Guiyang

Xi'an

Guangzhou

Zhaoqing

Weining

Xinyang

Jinan

Bortala Mongolian autonomous prefecture Bayingoleng Mongolian autonomous prefecture Alashankou

The amount of antibiotics detected (ng/L)

References

Sulfonamides Fluoroquinolones

$2.1 \sim 92.14$

$1.51 \sim 37.43$

$2.74 \sim 33.94$

$0 \sim 22.48$

32.42

$13.68 \sim 523.78$

ND 0.58

ND 478

$\leq 326.6$

1.3 117

ND 189.9

3.52 20.48

ND 0.81

ND 4.17

11.56 181.25

ND 247

13.68 523.78

$$
\text { 21.37 60 }
$$

687.90

9.27 190.71

50.5

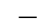

DN 196.5

22.50 103.72

ND 36.81

ND 61.01

$0.03 \sim 8.14$
$0.03 \sim 1.45$
$<2$
-
27.25

ND 267.0

ND 0.21

$14 \sim 474$

$\leq 36.5$

$-$

ND 148.7

70.70 155.52

$37.85 \sim 75.09$

49.49 83.31

ND 83.53

ND 16.4

$1.72 \sim 424.37$

4.7 64.28

814.1

2.27 9.46

43.2

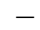

11.0 383.9

21.61 83.78

ND 99.32

293.78 5144.95

Macrolides

$0.26 \sim 18.07$

$0.18 \sim 4.46$

$0.61 \sim 33.77$

778.49

5.88 552.53

$0.04 \sim 0.94$

14 23

-

3.6 14.8

ND 18.48

$-$

$-$

$-$

$-$

19.1 223.7

5.88 552.53

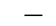

1112.2

ND 0.8

22.6

13.1 355. 6

13.16 309.09

$0.56 \sim 306.75$

$-$
Chloramphenicols Tetracyclines

-
-
-
-
46

6.46$$
-
$$$$
-
$$$$
-
$$$$
\text { 5 16.5 }
$$$$
-
$$$$
-
$$$$
-
$$$$
-
$$$$
-
$$$$
11.9 \sim 615.8
$$

$1.95 \sim 235.6$

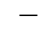

$-$

$-$

$-$

15.9

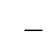

$-$

$-$

$-$

$-$

$-$
Wang et al. (2018c)

ND 12.19 Liang (2019)

0.70 14.07 Liang (2019)

9.98 177.28 Fang et al. (2017)

$14.55 \quad$ Feng et al. (2019)

- Wang

et al.,(2018d)

ND 4,720 Wu et at. (2016)

ND Xu et al. (2020)

ND 106.5 Ding (2018)

ND 14 Tang et al. (2014)

21.41 43.43 Xiao et al. (2019)

20.06 29.03 Xiao et al. (2019)

15.42 24.64 Xiao et al. (2019)

ND 18.08 Liu and Lu (2018)

- $\quad$ Feng et al. (2017)

0.36 243.25 Wang et al. (2018e)

4.64 129.91 Zhu et al. (2018)

643.2 Liu et al., 2017)

ND Xie et al. (2019)

ND Wang et al. (2020a)

ND 275.1 Wang and Wang (2020)

ND 8.91 Yan (2018)

ND 15.94 Wang (2020b)

ND 43.55 Yang (2018)

ND 12.01 Qiangian (2016) 
TABLE 3 | Antibiotic concentrations in surface water in aquaculture areas (From 2015 to 2020).

\begin{tabular}{|c|c|c|c|c|c|c|c|}
\hline \multirow[t]{2}{*}{ Waters } & \multirow[t]{2}{*}{ Regions } & \multicolumn{5}{|c|}{ The amount of antibiotics detected (ng/L) } & \multirow[t]{2}{*}{ References } \\
\hline & & Sulfonamides & Fluoroquinolones & Macrolides & Chloramphenicols & Tetracyclines & \\
\hline River estuary area & Dongying & $0.01 \sim 11.4$ & $0 \sim 63.3$ & ND 16.5 & $0.09 \sim 0.51$ & ND & Lian (2016) \\
\hline Gate of yang county & Yancheng & 1 2.35 & 3.77 9.76 & $0.15 \sim 2.05$ & $2.91 \sim 3.44$ & ND & Han et al. (2020) \\
\hline $\begin{array}{l}\text { Aquaculture ecosystem in } \\
\text { haiyang regions }\end{array}$ & Haiyang & $1.41 \sim 6.72$ & $2.14 \sim 180.14$ & $0.32 \sim 45.66$ & - & ND 2.09 & Chen et al. (2015) \\
\hline Marine aquaculture farms & Yangjiang & 1181.64 & 932.05 & 1158 & - & $>1536$ & $\begin{array}{l}\text { Zhang et al. } \\
(2018 b)\end{array}$ \\
\hline Maowei sea breeding pond & Guangxi province & 2.6 & 3 & 71.7 & 8.9 & - & $\begin{array}{l}\text { Zheng et al. } \\
(2017)\end{array}$ \\
\hline Freshwater aquaculture area & Zhejiang province & 73.6 171.1 & $94.1 \sim 113.2$ & ND & - & - & Xu et al. (2019) \\
\hline Aquaculture pond & Huzhou & ND 12,623 & ND 332.3 & - & - & ND 5.6 & $\begin{array}{l}\text { Wang et al. } \\
(2019)\end{array}$ \\
\hline Tilapia farming pond & Guangxi province & 44.3 & $14.7 \sim 22.7$ & - & ND & $32.7 \sim 3242$ & Yu et al.(2020) \\
\hline $\begin{array}{l}\text { Procambarus clarkii breeding } \\
\text { area }\end{array}$ & Jiangsu province & ND 198.827 & ND 38.82 & ND 38.471 & - & ND 114.296 & $\begin{array}{l}\text { Zhang et al. } \\
\text { (2019) }\end{array}$ \\
\hline Aquaculture area & Shanghai & ND 83.6 & ND 3.27 & - & - & ND 27.53 & $\begin{array}{l}\text { Zhong et al. } \\
(2018)\end{array}$ \\
\hline $\begin{array}{l}\text { Beijiang river and its surrounding } \\
\text { aquafarms }\end{array}$ & $\begin{array}{l}\text { Guangdong } \\
\text { province }\end{array}$ & 1016.4 & 480 & 375.8 & 210.5 & 277.5 & $\begin{array}{l}\text { Wang et al. } \\
\text { (2017b) }\end{array}$ \\
\hline Aquaculture ponds & Honghu & 32 1096.8 & $18 \sim 334.3$ & - & - & $204.1 \sim 3028.3$ & Yuan et al. (2019) \\
\hline Hangzhou bay & Zhejiang province & $16.6 \sim 44.13$ & $41.11 \sim 115.28$ & - & $8.41-28.57$ & $25.98 \sim 50.2$ & Hao et al. (2017) \\
\hline Nansha aquaculture area & Guangzhou & 0.81 & ND & 78.01 & - & - & Chen et al. (2018) \\
\hline Panyu aquaculture area & Guangzhou & $113 \sim 247$ & $0 \sim 100$ & $80 \sim 1400$ & - & - & Lian (2016) \\
\hline
\end{tabular}

TABLE 4 | Antibiotic concentrations in drinking water sources in some cities (From 2015 to 2020).

\begin{tabular}{|c|c|c|c|c|c|c|c|}
\hline \multirow[t]{2}{*}{ Waters } & \multirow[t]{2}{*}{ Regions } & \multicolumn{5}{|c|}{ The amount of antibiotics detected (ng/L) } & \multirow[t]{2}{*}{ References } \\
\hline & & Sulfonamides & Fluoroquinolones & Macrolides & Chloramphenicols & Tetracyclines & \\
\hline Huizhou water supply plant & Huizhou & 47.73 & ND & 11.65 & - & ND & Zheng (2017) \\
\hline Beijing groundwater & Beijing & ND 341.2 & ND 49.5 & - & - & ND 3.2 & Chen et al. (2017) \\
\hline Drinking water in qingyuan & Qingyuan & ND 62.63 & ND 295.09 & ND 9.94 & ND & ND & Dai (2019) \\
\hline Biliuhe reservoir & Dalian & ND 15 & ND 130 & ND & ND 17 & - & Dong et al. (2020) \\
\hline Lianhua reservoir & Xiamen & ND 11.96 & ND 353.42 & ND 305.19 & - & ND 254.67 & Liao et al. (2020) \\
\hline Drinking water sources & Dongguan & $24.63 \sim 38.7$ & $11.11 \sim 82.24$ & $27.25 \sim 206.65$ & - & $20.08 \sim 43.02$ & Xie et al. (2020) \\
\hline Reservoir & Chongqing & ND 40.2 & - & ND 60.1 & $1.1 \sim 35.4$ & - & Feng et al. (2020) \\
\hline Water plant & Suzhou & 4.65 & 2.29 & 1.51 & - & 1.45 & Di et al. (2019) \\
\hline Drinking water sources & Nanjing & ND 29.58 & ND 40.18 & $0.53 \sim 35.25$ & - & ND 35.61 & Liu et al. (2020a) \\
\hline Drinking water sources & Jiaxing & $0.69 \sim 13.21$ & 89.5 230.8 & ND 56.9 & $121 \sim 259$ & - & Guo et al. (2016) \\
\hline Taipu river and jinze reservoir & Shanghai & 10.5 385.8 & 10.2 189.4 & ND 56.8 & - & $8.1 \sim 135.5$ & Li et al. (2020a) \\
\hline Fengshuba reservoir & $\begin{array}{l}\text { Guangdong } \\
\text { province }\end{array}$ & 4.29 39.45 & ND 302.76 & ND 1.57 & - & $3.44 \sim 345$ & Chen et al. (2020b) \\
\hline
\end{tabular}

regulations of the above industries is the main approach to reduce antibiotic discharge.

\section{Antibiotics in Sediment}

Antibiotics have been detected not only in water bodies such as lakes but also in sediments, which can contain significant amounts. The level of exposure of sediments to antibiotics is usually higher than that of water because the sediment particles, which have a strong ability to adsorb antibiotics (Lee and Carlson, 2006; Kim and Carlson, 2007; ; Yang et al., 2010). Antibiotic concentrations in surface water are more susceptible to external environmental influences than those in sediment, including dilution (Cheng et al., 2014; Ding et al., 2017), adsorption of particles (Wang et al., 2017a; Yang et al., 2020), and photodegradation (Chen et al., 2016), all of which can affect the variation of the antibiotic concentrations in water. Compared to in the water column, antibiotic levels in sediments are relatively stable because their ability to strongly adsorb antibiotics leads to antibiotic accumulation in the sediments (Mangalgiri and Blaney, 2017). Different water environments lead to different adsorption properties of the sediments, resulting in a both spatially and geographically heterogeneous distribution of antibiotics in sediments, as shown in Table 5. Furthermore, this distribution may also be influenced by the external environment. For example, external currents may flush antibiotics-bearing sediments and thus release adsorbed antibiotics into the aquatic environment, causing secondary pollution (Radke et al., 2009). Sediments can affect the level of antibiotics in water bodies. The components of sediments are highly complex, and there are several differences in the composition of sediments in different water body 


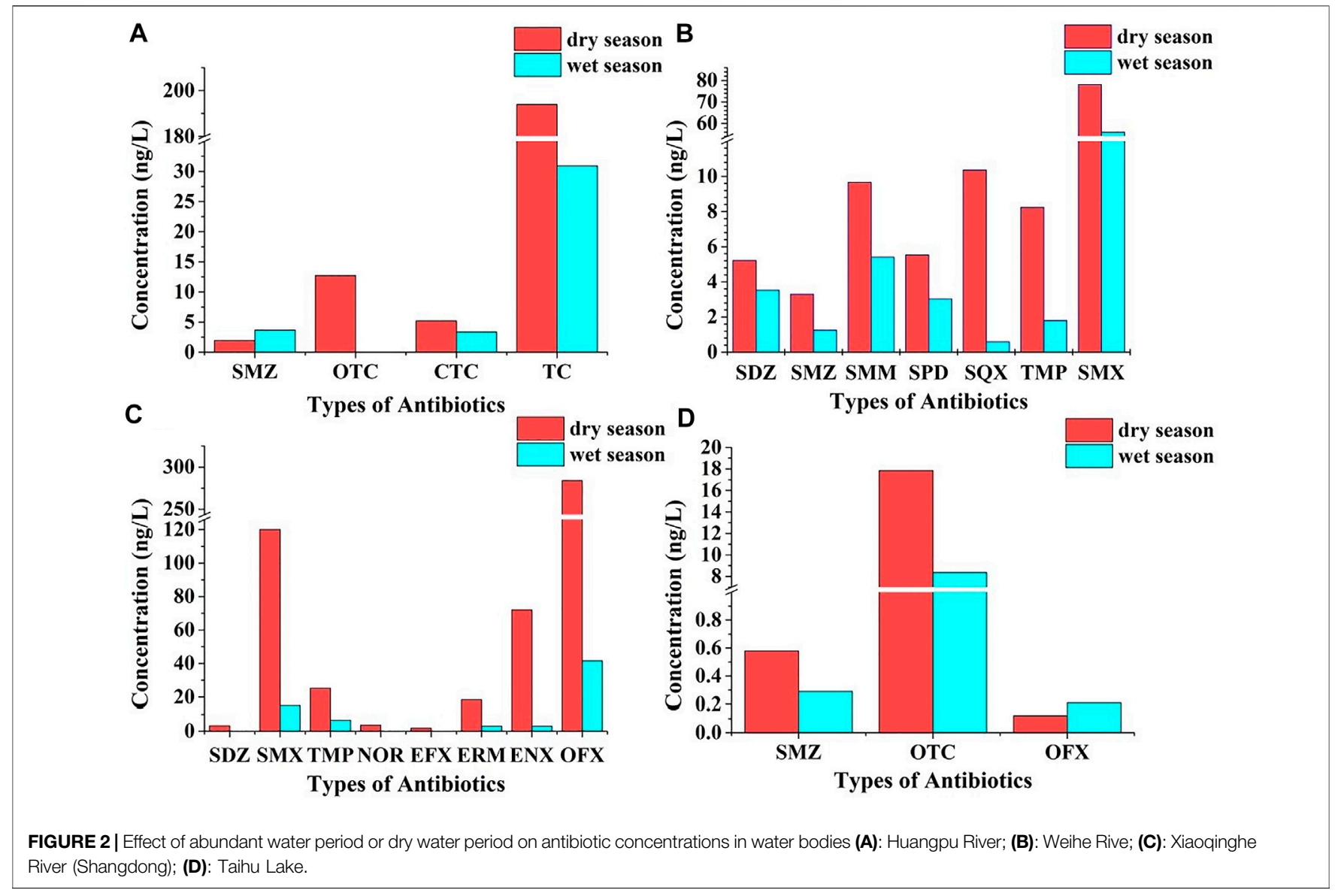

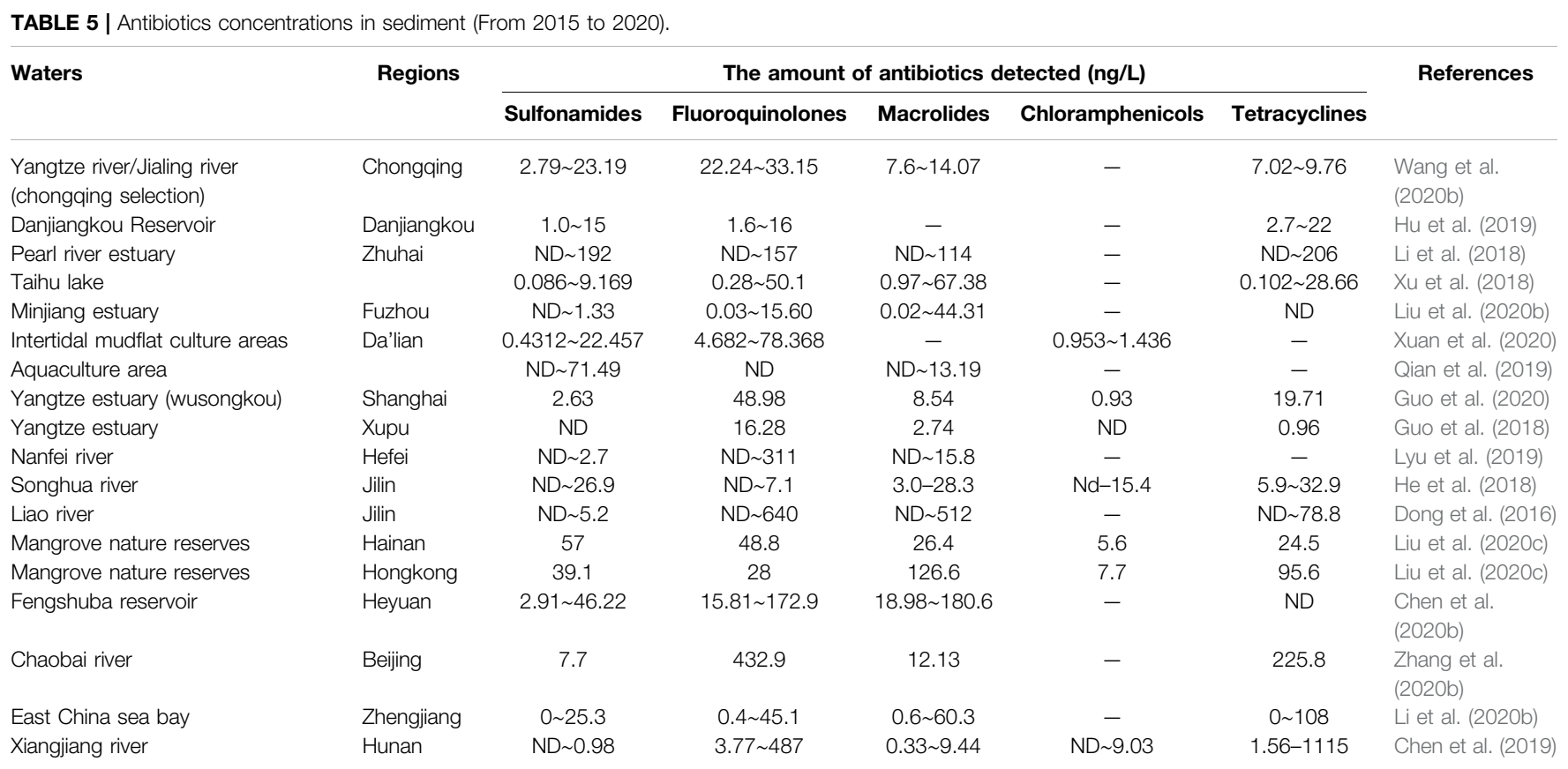



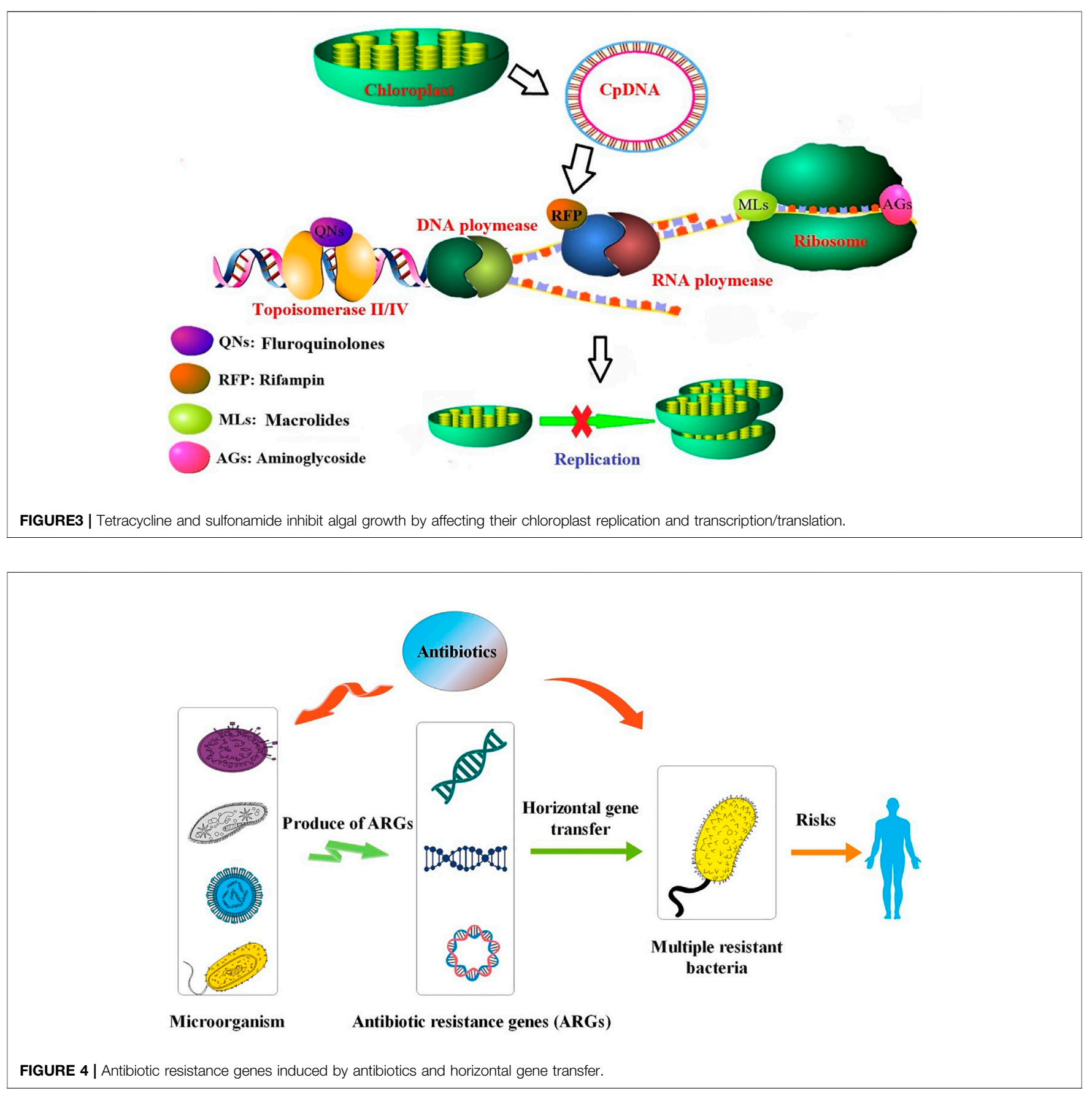

environments. The enrichment of antibiotics in sediments and how antibiotics in sediments are released into the water body still need systematic and in-depth research.

\section{EFFECT OF ANTIBIOTICS ON PEOPLE AND ECOSYSTEMS}

\section{Antibiotic Hazards to Human Health}

Antibiotics have been found in fish from some farming areas and in some cooked foods and crops, and the antibiotics can be enriched in humans after consumption. Antibiotics have a strong inhibitory effect on the entire intestinal bacterial community (He et al., 2014). Antibiotics have a strong inhibitory effect on the entire bacterial community of intestinal microorganisms. The transfer of resistance genes between intestinal endophytes and pathogenic bacteria such as Escherichia coli, Klebsiella, and Enterococcus faecalis leads to an imbalance in intestinal microorganisms, and this in turn causes a variety of bacterial diseases (McInnes et al., 2020) and even intestinal cancer (Sobhani et al., 2019) and experiments have shown that even a small amount of antibiotics rapidly 

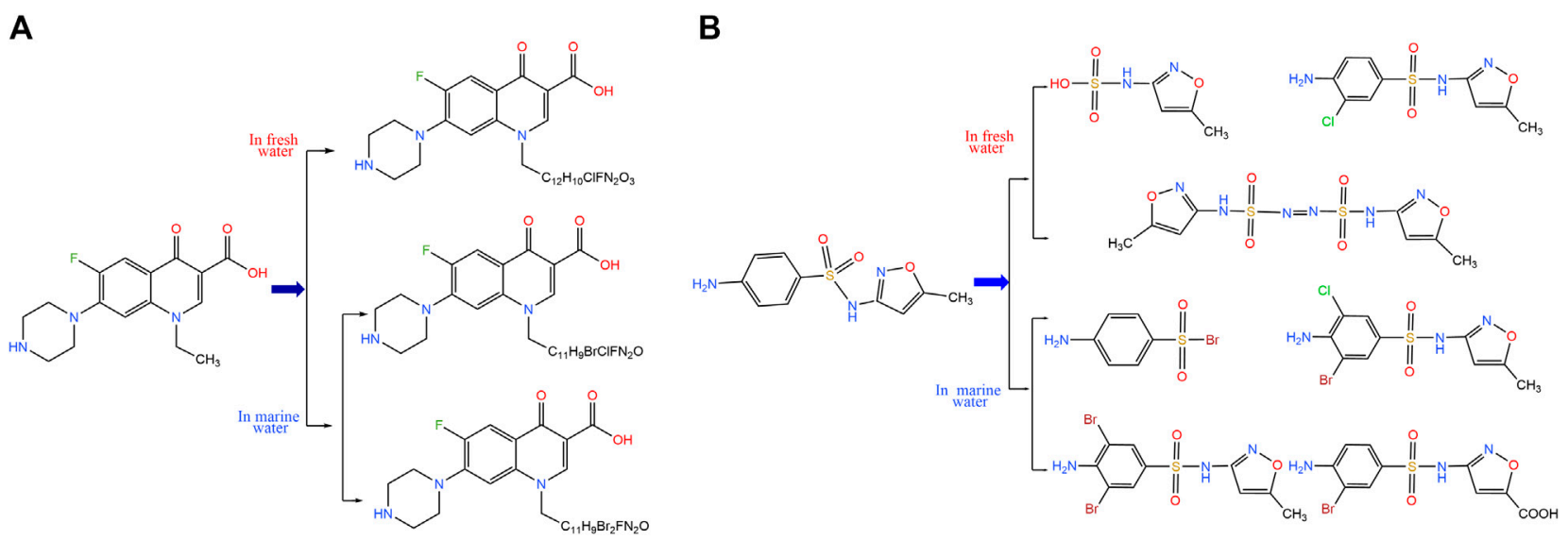

FIGURE 5 | Conversion of antibiotics to disinfection byproducts. End products of Norfloxacin or Sulfamethoxazole (Mclnnes et al.) reacts with NaClO in different water environments.

TABLE 6 | Characteristics of different methods of degrading antibiotics.

\begin{tabular}{|c|c|c|c|c|}
\hline Methods of degrading antibiotics & Stages of application & Advantages & Disadvantages & References \\
\hline Physical adsorption & $\begin{array}{l}\text { Scaled up applications in } \\
\text { industry }\end{array}$ & $\begin{array}{l}\text { Low cost, simple design, and high } \\
\text { flexibility }\end{array}$ & $\begin{array}{l}\text { Low efficiency and causes } \\
\text { secondary pollution }\end{array}$ & Dutta and Mala (2020) \\
\hline Membrane filtration & $\begin{array}{l}\text { Applications in drinking water } \\
\text { production }\end{array}$ & Removal of many contaminants & High cost & Yin et al. (2021) \\
\hline Chemical oxidative degradation & $\begin{array}{l}\text { Scaled up applications in } \\
\text { industry }\end{array}$ & $\begin{array}{l}\text { Easy to operate, requires short time, } \\
\text { and efficient }\end{array}$ & $\begin{array}{l}\text { Environmentally unfriendly } \\
\text { and low recycling rate }\end{array}$ & $\begin{array}{l}\text { (Advanced oxidation } \\
\text { technology engineering. }\end{array}$ \\
\hline Photolysis & In the laboratory research stage & Eco-friendly & High cost & Bo (2017) \\
\hline Plant adsorption & $\begin{array}{l}\text { Scaled up applications in } \\
\text { industry }\end{array}$ & Economical, with high efficiency & $\begin{array}{l}\text { Selective and diffusion of } \\
\text { resistance genes }\end{array}$ & Liu et al. (2019) \\
\hline $\begin{array}{l}\text { Activated sludge and microbial } \\
\text { strains }\end{array}$ & $\begin{array}{l}\text { Scaled up applications in } \\
\text { industry }\end{array}$ & Efficient and easy to operate & $\begin{array}{l}\text { Susceptible to microbial } \\
\text { resistance gene production }\end{array}$ & Liu et al. (2016) \\
\hline Enzymatic degradation & In the laboratory research stage & $\begin{array}{l}\text { Eco-friendly, efficient, and requires } \\
\text { short time }\end{array}$ & $\begin{array}{l}\text { Highly influenced by } \\
\text { environment }\end{array}$ & Bilal et al. (2019) \\
\hline
\end{tabular}

changes the diversity of the intestinal flora in a short period of time (Dethlefsen et al., 2007; Fouhy et al., 2012), which may lead to a variety of diseases, especially in newborns. Antibiotic use during pregnancy or in newborns may adversely affect the neonatal gut microbiome and adversely affect the development of the infant's immune system, leading to childhood atopy, asthma, allergies, and obesity. It increases the probability of epilepsy in children (Kenyon et al., 2008; Neu and Walker, 2011; Madan et al., 2012; Mette et al., 2012; Dik et al., 2016; Koebnick et al., 2019; Milliken et al., 2019; Pronovost and Hsiao, 2019; Tadeusz et al., 2019; Zimmermann and Curtis, 2020; Zhang et al., 2021). Excessive intake of antibiotics can cause damage to the nervous system, kidneys, and other organs (Ramirez et al., 2007). At the same time, germs are prone to develop drug resistance and become super germs that are difficult to cure (Goldman, 2004; Xu et al., 2010). The dose of antibiotics used to treat a disease is controllable, but the enrichment of antibiotics from food into the body is not measurable and assessable. As food is consumed every day, determining the content of antibiotics contained in food is difficult. Therefore, the regulation of antibiotic content in food in the market is important.

\section{Antibiotic Accumulation in the Ecological Chain}

Algae are the basis of the food chain, and even a slight decrease in algal populations may affect the balance of the aquatic system (Lanzky and Halling-Sørensen, 1998). Researchers performed relevant cytotoxicity experiments to verify that the presence of antibiotics affects the genetic and normal growth of the genome (Yamaguchi et al., 2003; Yamaguchi and Subramanian, 2003). As shown in Figure 3, tetracycline and sulfonamide antibiotics have been found to inhibit algal growth by affecting their chloroplast 


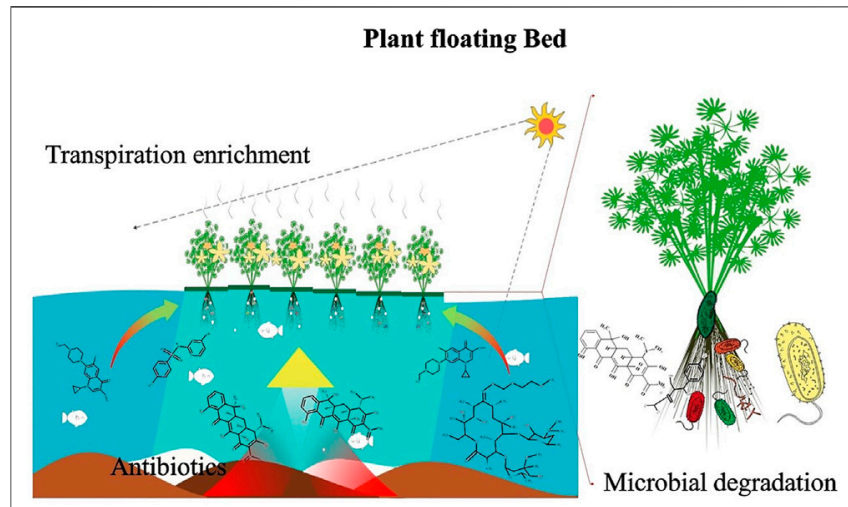

FIGURE 6 | Schematic diagram of plant floating bed adsorption of antibiotics. Plant roots adsorb antibiotics in the aquatic environment and sediment, and then antibiotics are degraded by microorganisms enriched by the roots.

replication, transcription/translation, and metabolic pathways (Brain et al., 2004; Brain et al., 2008; Baran et al., 2011). Fish appear to be less sensitive to antibiotics than algae (Li et al., 2012a). Feeding habits can affect the accumulation of antibiotics in fish, and some studies have shown that carnivorous fish have higher levels of antibiotic enrichment than other fish because they are the top consumers in the food chain in the aquatic environment ( $\mathrm{He}$ et al., 2014). Zhao et al. showed that the accumulation of antibiotics in different tissues of animals is different, and the accumulated antibiotic levels in different tissues of fish are also different. For example, the accumulation levels of antibiotics in fish bile, plasma, and liver are relatively higher than those in other organs. (Zhao et al., 2016a). Invertebrates and fish that are chronically exposed to antibiotics are enriched in antibiotics, and people who consume these aquatic organisms face a high health risk (Metsälä et al., 2015; Wang et al., 2015; Möhle et al., 2016; Siswanto et al., 2016; Winek et al., 2016). The toxicity of antibiotics in water is influenced by their concentration, duration of exposure, aquatic species, and the coexistence of other antibiotics and/or other contaminants (Grenni et al., 2017). Plants can take up multiple antibiotics from soil and water, and while the toxicities of these multiple antibiotics are not superimposed on a single toxicity, they can induce combined toxicity (Brain et al., 2004). Antibiotics are passed up the food chain, resulting in human exposure to antibiotics via the consumption of food containing antibiotics; eventually, the accumulated antibiotics will have negative effects on the human body.

\section{Increase of Potential Novel Antibiotic Resistant Gene Induction by Antibiotics}

Genes are mutated and inherited in nature, and antibiotic resistance genes (ARGs) are present in the natural environment. Thus, antibiotics released into the environment exert selective pressure on the microbial community, thereby inducing drug-resistant bacteria and causing widespread bacterial resistance (Wei et al., 2019). Residual antibiotics and ARGs that enter the environment can be taken up by plants and enter the food chain through the migration distribution of the soil-water plant system (Forsberg et al., 2012), where they migrate and accumulate, and eventually enter the human body. There are two main sources of ARGs in the environment (Zhang et al., 2019a): ARGs are present in the environment itself-Antibiotics are mainly derived from secondary metabolites of microorganisms, plants, and animals, and these microorganisms are resistant to the antibiotics they produce (Russell and Yost, 2020); 2) Another source of ARGs is exogenous input (Zhang et al., 2018a). The metabolism rate of antibiotics in animals is very low, and about $25-75 \%$ of the antibiotics enter the environment through excreta without having been metabolized, thus inducing the production of resistance genes in the environment. As illustrated in Figure 4, ARGs can be transmitted between microorganisms and vertically between generations through horizontal gene transfer (HGT). As microorganisms evolve, new ARGs may also be produced (Ji et al., 2012; Shi et al., 2015). A wide variety of microorganisms in the environment may even lead to the creation of multidrug resistance genes or superbugs, while also increasing the potential for the induction of novel ARGs. The food chain can enhance the spread of resistance genes (Hu et al., 2016; Johnson et al., 2016). The transfer of ARGs acquired by humans from the environment or from food to gut microbes leads to an increase in gut microbial resistance (Huddleston, 2014), Studies have identified $\beta$-lactamase genes in the metabolic genome of human gut flora (Cao et al., 2021), suggesting that human gut microbes can acquire resistance genes from the environment, thereby leading to drug-resistant infections in human (Bengtsson-Palme, 2017).

\section{Antibiotics and Disinfection Byproducts}

In addition to their own toxicological effects and the genetic problem of resistance, antibiotics that remain in the aquatic environment have been found by researchers to be precursors of disinfection byproducts (DBPs) that can react with disinfectants such as chlorine and chlorine dioxide to produce halogenated carbon or nitrogen-containing disinfection byproducts (Wang and Helbling 2016; Zhang et al., 2017; Chuan et al., 2018) (Figure 5). DBPs are the result of the reaction between disinfectants and a special class of organics produced by the reaction of organic precursors in water. Since many antibiotics are nitrogen-containing organics, they contribute significantly to some of the more toxic N-DBPs. These disinfection byproducts, in turn, can be instrumental in inducing antibiotic resistance and resistance genes. DBPs have been shown to significantly increase bacterial resistance to antibiotics (Lü et al., 2015) and the mutagenesis rate of resistance genes ( $\mathrm{Li}$ et al., 2016) as well as the concentration of resistant bacteria ( $\mathrm{Lv}$ et al., 2015). The level of DBPs is often overlooked when testing for antibiotics in water, and we need to pay equal attention to the level of DBPs, which can also lead to the development of resistance genes in bacteria.

\section{Antibiotics in Aquatic Organisms}

Low concentrations of antibiotics are present in fish; however, they are mainly detected in laboratory studies. The 
TABLE 7 | Antibiotic adsorption by plants reported in the literature.

\begin{tabular}{|c|c|c|c|c|}
\hline Antibiotics & & Plant & Removal (\%) & References \\
\hline \multirow[t]{24}{*}{ Sulfonamides } & Sulfadiazine & Thalia & $72 \pm 6$ & Dan et al. (2013) \\
\hline & Sulfadiazine & Arundo & $74 \pm 6$ & Dan et al. (2013) \\
\hline & Sulfadiazine & Waseyutaka & 98.7 & Xian et al. (2010) \\
\hline & Sulfadiazine & Acorus calamus & $26.42-64.93$ & Chen et al. (2020a) \\
\hline & Sulfamethoxazole & Cyperus alternifolius & $76.77 \pm 4.13$ & Kumar et al. (2005) \\
\hline & Sulfamethoxazole & Dryan & 91.8 & Xian et al. (2010) \\
\hline & Sulfamethoxazole & T. angustifolia/P. australis & $60 \pm 26$ & Hijosa-Valsero et al. (2011a) \\
\hline & Sulfamethoxazole & Acorus/Typha & $29-52$ & Park et al. (2009) \\
\hline & Sulfamethoxazole & Thalia Dealbata Fraser & $56.9 \pm 7.36$ & Jun et al. (2016) \\
\hline & Sulfamethoxazole & Iris tectorum Maxim & $69.2 \pm 2.24$ & Jun et al. (2016) \\
\hline & Sulfamethoxazole & T. angustifolia & $80 \pm 42$ & Hijosa-Valsero et al. (2011b) \\
\hline & Sulfamethoxazole & Acorus calamus & $37.81-84.05$ & Chen et al. (2020a) \\
\hline & Sulfamethazine & Waseyutaka & 88.8 & Xian et al. (2010) \\
\hline & Sulfamethazine & Tachimasari & 89.4 & Xian et al. (2010) \\
\hline & Sulfamethazine & H. pennisetum & $91-95$ & Liu et al. (2013) \\
\hline & Sulfamethazine & Thalia Dealbata Fraser & $100 \pm 0.00$ & Jun et al. (2016) \\
\hline & Sulfamethazine & Iris tectorum Maxim & $100 \pm 0.00$ & Jun et al. (2016) \\
\hline & Sulfamethazine & Acorus calamus & $18.98-65.06$ & Chen et al. (2020a) \\
\hline & Sulfadimethoxine & T. angustifolia & $99 \pm 2$ & Hijosa-Valsero et al. (2011b) \\
\hline & Sulfadimethoxine & P. australis & $99 \pm 2$ & Hijosa-Valsero et al. (2011b) \\
\hline & Trimethoprim & Thalia & $31 \pm 37$ & Dan et al. (2013) \\
\hline & Trimethoprim & Arundo & $38 \pm 10$ & Dan et al. (2013) \\
\hline & Trimethoprim & Thalia Dealbata Fraser & $84.6 \pm 2.26$ & Jun et al. (2016) \\
\hline & Trimethoprim & Iris tectorum Maxim & $84.4 \pm 2.28$ & Jun et al. (2016) \\
\hline \multirow[t]{6}{*}{ Lincosamides } & Roxithromycin & Cyperus alternifolius & $85.35 \pm 2.47$ & Kumar et al. (2005) \\
\hline & Leucomycin & Thalia Dealbata Fraser & $100 \pm 0.00$ & Jun et al. (2016) \\
\hline & Leucomycin & Iris tectorum Maxim & $100 \pm 0.00$ & Jun et al. (2016) \\
\hline & Clarithromycin & Thalia Dealbata Fraser & $74.2 \pm 5.63$ & Jun et al. (2016) \\
\hline & Clarithromycin & Iris tectorum Maxim & $87.3 \pm 0.86$ & Jun et al. (2016) \\
\hline & Erythromycin & P. australis & $64 \pm 30$ & Hijosa-Valsero et al. (2011b) \\
\hline \multirow[t]{11}{*}{ Tetracyclines } & Oxytetracycline $\mathrm{HCl}$ & H. pennisetum & $68-73$ & Liu et al. (2013) \\
\hline & Oxytetracycline & P. australis & $92.7-99.9$ & Huang et al. (2015) \\
\hline & Oxytetracycline & H. annuus & 100 & Gujarathi et al. (2005) \\
\hline & Oxytetracycline & Typha orientalis & 72.43 & Zhao et al. (2016b) \\
\hline & Chlorotetracycli & P. australis & $69.0-99.7$ & Huang et al. (2015) \\
\hline & Chlorotetracycli & Typha orientalis & 73.84 & Zhao et al. (2016b) \\
\hline & Tetracycline & $P$. australis & 88.4-98.3 & Huang et al. (2015) \\
\hline & Tetracycline & P. australis & $99.3 \pm 0.4$ & Fernandes et al. (2015) \\
\hline & Tetracycline & Typha orientalis & 70.85 & Zhao et al. (2016b) \\
\hline & Tetracycline & H. annuus & 100 & Gujarathi et al. (2005) \\
\hline & Doxycycline & T. angustifolia & $75 \pm 40$ & Hijosa-Valsero et al. (2011b) \\
\hline \multirow[t]{3}{*}{ Fluroquinolones } & Ciprofloxacin $\mathrm{HCl}$ & H. pennisetum & $78-84$ & Liu et al. (2013) \\
\hline & Enrofloxacin & P. australis & $98.7 \pm 0.3$ & Fernandes et al. (2015) \\
\hline & Difloxacin & Phragmites australis & $>84$ & Jun et al. (2016) \\
\hline \multirow[t]{6}{*}{ Other PPCPs } & Naproxen & Acorus/Typha & $52-92$ & Park et al. (2009) \\
\hline & Naproxen & Phragmites australis & $80 \pm 9$ & Matamoros and Bayona (2006) \\
\hline & Ibuprofen & Phragmites australis & $62 \pm 2$ & Matamoros and Bayona (2006) \\
\hline & Diclofenac & Acorus/Typha & $73-96$ & Park et al. (2009) \\
\hline & Carbamazepine & Acorus/Typha & $30-47$ & Park et al. (2009) \\
\hline & Methyl dihydrojasmonate & Phragmites australis & $99 \pm 1$ & Matamoros and Bayona (2006) \\
\hline
\end{tabular}

concentrations of antibiotics in aquatic organisms are correlated with their habits, their position in the food chain, and vary in fish and shrimp from different pelagic layers, depending on their location in the aquatic environment (Li et al., 2012b). In a previous study, sediments adsorbed with antibiotics were collected and used to construct an ecosystem to cultivate zebrafish (Chen et al., 2017a). The presence of antibiotics was detected in the zebrafish, suggesting bioconcentration of antibiotics in aquatic organisms Fish in wild water environments were tested for antibiotic levels and antibiotic levels increased progressively from herbivorous to omnivorous to carnivorous, possibly via food chain enrichment (Tang et al., 2020). Antibiotics with different properties showed tissue specificity in aquatic products, suggesting significant differences in bioaccumulation factors between antibiotics (Liu et al., 2014; Zhao et al., 2015; Zhao et al., 2016a). Bioaccumulation and the different metabolic pathways of different aquatic organisms lead to a more complex accumulation of antibiotics in aquatic organisms. With the improvement in living standards, there is an increasing demand for aquatic products. The 
TABLE 8 | Antibiotic-degrading bacterial strains reported in the recent three years.

\begin{tabular}{|c|c|c|c|c|c|c|}
\hline Antibiotics & & Bacterial species & $\begin{array}{c}\text { Concentration } \\
(\mathrm{mg} / \mathrm{L})\end{array}$ & $\begin{array}{c}\text { Removal rate } \\
(\%)\end{array}$ & Sources & References \\
\hline$\beta$-lactam & Cephalosporin & Achromobacter sp. YF-1 & 30 & 92.71 (7 days) & Waste medicine residue & Zhang et al. (2019a) \\
\hline \multirow[t]{12}{*}{ Sulfonamides } & Sulfamethoxazole & $\begin{array}{l}\text { Shewanella oneidensis } \\
\text { MR-1 }\end{array}$ & 10 & 97.9 (3 days) & Laboratory & Mao et al. (2018) \\
\hline & Sulfamethoxazole & Shewanella sp. MR-4 & 10 & 100 (3 days) & Laboratory & Mao et al. (2018) \\
\hline & Sulfamethoxazole & $\begin{array}{l}\text { Pseudomonas mandelii } \\
\text { McBPA4 }\end{array}$ & 50 & 73 (15 days) & Laboratory & Reis et al. (2018) \\
\hline & Sulfamethoxazole & Acinetobacter sp. W1 & $5 \sim 240$ & $100(10 \mathrm{~h})$ & Activated sludge & $\begin{array}{l}\text { Wang and Wang } \\
\text { (2018) }\end{array}$ \\
\hline & Sulfamethoxazole & $\begin{array}{l}\text { Ochrobactrum sp. SMX- } \\
\text { PM1-SA1 }\end{array}$ & 5 & 45.2 (8 days) & Wastewater & Zhang et al. (2019c) \\
\hline & Sulfamethoxazole & Labrys sp. SMX-W11 & 5 & 62.2 (8 days) & Activated sludge & Mulla et al. (2018) \\
\hline & Sulfamethoxazole & $\begin{array}{l}\text { Gordonia sp. SMX-W2- } \\
\text { SCD14 }\end{array}$ & 5 & 51.4 (8 days) & Pig manure & Mulla et al. (2018) \\
\hline & Sulfamethoxazole & Achromobacter sp. JL9 & 50 & $79.45(120 \mathrm{~h})$ & $\begin{array}{l}\text { Pharmaceutical } \\
\text { wastewater }\end{array}$ & Liang et al. (2019) \\
\hline & Sulfamethoxazole & Acinetobacter sp & 30 & $100(5 \mathrm{~h})$ & $\begin{array}{l}\text { Microbiological culture } \\
\text { collection }\end{array}$ & Wang et al. (2018a) \\
\hline & Sulfadimidine & Bacillus cereus J2 & 50 & 100 (36 h) & Municipal sewage & Zhang et al. (2019c) \\
\hline & Sulfamethazine & Acinetobacter sp & 30 & $100(10 \mathrm{~h})$ & $\begin{array}{l}\text { Microbiological culture } \\
\text { collection }\end{array}$ & Wang et al. (2018a) \\
\hline & Sulfadiazine & Acinetobacter sp & 30 & $100(10 \mathrm{~h})$ & $\begin{array}{l}\text { Microbiological culture } \\
\text { collection }\end{array}$ & Wang et al. (2018a) \\
\hline \multirow[t]{7}{*}{ Tetracyclines } & Tetracycline & Raoultella sp. XY-1 & 20 & 70.68 (8 days) & Pig farm sediment & Wu et al. (2018) \\
\hline & Tetracycline & Klebsiella sp. SQY5 & $10 \sim 30$ & 67.32 (92 h) & Municipal sludge & Shao et al. (2018a) \\
\hline & Tetracycline & Achromobacter sp. TJ-2\# & 50 & 63.9 (3 days) & Contaminated soil & Cheng et al. (2017b) \\
\hline & Oxytetracycline & Pseudomonas sp. T4 & 100 & 26.88 (7 days) & Livestock manure & Meng et al. (2018) \\
\hline & Oxytetracycline & Achromobacter sp. TJ-2\# & 50 & 58.3 (3 days) & Contaminated soil & Cheng et al. (2017b) \\
\hline & Oxytetracycline & Ochrobactrum sp. KSS10 & 30 & 63.33 (4 days) & Municipal sludge & Shao et al. (2018b) \\
\hline & Aureomycin & Achromobacter sp. TJ-2\# & 50 & 65.5 (3 days) & Contaminated soil & Cheng et al. (2017b) \\
\hline \multirow[t]{8}{*}{ Fluoroquinolones } & Norfloxacin & $\begin{array}{l}\text { Staphylococcus caprae } \\
\text { NOR-36 }\end{array}$ & 5 & 92.6 (10 days) & $\begin{array}{l}\text { Pharmaceutical } \\
\text { wastewater }\end{array}$ & Fu et al. (2017) \\
\hline & Ofloxain & Labrys portucalensis F11 & 0.45 & 34.6 (28 days) & Fluorobenzene & (Maia et al., 2018) \\
\hline & Ofloxain & Rhodococcus sp. FR1 & 0.45 & 39.3 (28 days) & Fluorobenzene & (Maia et al., 2018) \\
\hline & Ciprofloxacin & Thermus thermophiles C419 & 5 & 57 (5 days) & Pharmaceutical sludge & (Pan et al., 2017) \\
\hline & Ciprofloxacin & $\begin{array}{l}\text { Enterobacter sp. } \\
\text { (KM504128) }\end{array}$ & 5 & 96 (14 days) & Hospital effluent water & $\begin{array}{l}\text { Liyanage and Manage } \\
\text { (2018) }\end{array}$ \\
\hline & Ciprofloxacin & $\begin{array}{l}\text { Lactobacillus gesseri. } \\
\text { (KM4055978) }\end{array}$ & 5 & 100 (14 days) & Hospital effluent water & $\begin{array}{l}\text { Liyanage and Manage } \\
\text { (2018) }\end{array}$ \\
\hline & Ciprofloxacin & Bacillus sp. (KM504129) & 5 & 74 (14 days) & Hospital effluent water & $\begin{array}{l}\text { Liyanage and Manage } \\
\text { (2018) }\end{array}$ \\
\hline & Ciprofloxacin & Bradyrhizobium sp. GLC_01 & 0.05 & $70.4 \pm 7.4$ (8 days) & Wastewater & Nguyen et al. (2018) \\
\hline Macrolides & Tylosin & Achromobacter & 50 & 96.08 (56 days) & Spinach soil & Zhang et al. (2018c) \\
\hline
\end{tabular}

government should strengthen the regulation of antibiotic content in aquatic products. There is also a need to strengthen the regulation of fishery drugs and scientific use of drugs and improve the code of practice and standards.

\section{DEGRADATION OF ANTIBIOTICS}

Antibiotic pollution is becoming increasingly serious globally. Although countries with severe antibiotic pollution have introduced corresponding policies, they have come too late and/or are inadequate to solve the problem (Kara, 2019). Currently, researchers are in the process of developing environmentally friendly alternatives to antibiotics to reduce the use of antibiotics. At the same time, owing to the excessive antibiotic content in the aquatic environment, researchers have developed various antibiotic treatment techniques to degrade antibiotics. These methods can be roughly divided into the following categories: physical adsorption, chemical oxidation, photodegradation, and biodegradation. The general characteristics of various methods of removing antibiotics from water bodies are shown in Table 6.

\section{Physical Removal of Antibiotics}

The removal of antibiotics from the aquatic environment can be achieved by adsorbing the antibiotics on an adsorbent and then recovering the adsorbent loaded with antibiotics. Existing physical methods include physical adsorption, membrane filtration, and precipitation. However, the physical methods can only separate the antibiotics from the environment but not degrade them, and subsequent treatment is necessary. 
TABLE 9 | Antibiotic-degrading fungi reported in the recent 5 years.

\begin{tabular}{|c|c|c|c|c|c|c|}
\hline Antibiotics & & Fungus & $\begin{array}{c}\text { Concentration } \\
(\mathrm{mg} / \mathrm{L})\end{array}$ & $\begin{array}{c}\text { Removal rate } \\
\text { (\%) }\end{array}$ & $\begin{array}{l}\text { Degradation } \\
\text { enzyme }\end{array}$ & References \\
\hline \multirow[t]{7}{*}{ Sulfonamides } & Sulfadiazine & Fusarium solani KS256 & 1.5 & 18.53 (7 days) & - & Wang et al. (2018b) \\
\hline & Sulfamethoxazole & Pycnoporus sanguineus & 10 & 100 (2 days) & Lac P450 & Gao et al. (2017a) \\
\hline & Sulfamethoxazole & Phanerochaete Chrysosporium & 10 & 63.3 (8 days) & MnP and LiP P450 & Gao et al. (2017a) \\
\hline & Sulfamethoxazole & Phoma sp. UHH5-1-03 & 10 & 57 (168 h) & Lac & $\begin{array}{l}\text { Hofmann and Schlosser } \\
\text { (2016) }\end{array}$ \\
\hline & Sulfamethoxazole & $\begin{array}{l}\text { Trametes versicolor ATCC } \\
42530\end{array}$ & 10 & 90-94 (30 days) & - & Aydin (2016) \\
\hline & Sulfamethoxazole & $\begin{array}{l}\text { Bjerkandera adusta ATCC } \\
28314\end{array}$ & 10 & 90-94 (30 days) & - & Aydin (2016) \\
\hline & Sulfamethoxazole & $\begin{array}{l}\text { Achromobacter } \\
\text { denitrificans strain PR1 }\end{array}$ & 150 & $91(51 \mathrm{~h})$ & - & Nguyen et al. (2018) \\
\hline \multirow[t]{11}{*}{ Fluoroquinolones } & Ciprofloxacin & Pleurotus ostreatus & 500 & 95.07 & $\mathrm{MnP}$ & Singh et al. (2017) \\
\hline & Norfloxacin & Penicillium janthinellum KS272 & 1.5 & 10.59 (8 days) & - & Wang et al. (2018b) \\
\hline & Ciprofloxacin & Pycnoporus sanguineus & 10 & 98.5 (2 days) & Lac P450 & Gao et al. (2017a) \\
\hline & Norfloxacin & Pycnoporus sanguineus & 10 & 96.4 (2 days) & Lac P450 & Gao et al. (2017a) \\
\hline & Ciprofloxacin & Phanerochaete Chrysosporium & 10 & 64.5 (8 days) & MnP and LiP P450 & Gao et al. (2017a) \\
\hline & Norfloxacin & Phanerochaete Chrysosporium & 10 & 73.2 (8 days) & MnP and LiP P450 & Gao et al. (2017a) \\
\hline & Norfloxacin & Irpex lacteus & 10 & 100 (10 days) & Lac P450 & Čvančarová et al. (2015) \\
\hline & Norfloxacin & Trametes versicolor & 10 & 85 (14 days) & $\mathrm{MnP}$ and MIP & Čvančarová et al. (2015) \\
\hline & Ofloxacin & Irpex lacteus & 10 & 100 (10 days) & $\mathrm{MnP}$ and MIP & Čvančarová et al. (2015) \\
\hline & Ciprofloxacin & Irpex lacteus & 10 & 100 (10 days) & $\begin{array}{l}\text { Lac and MnP } \\
\text { and MIP }\end{array}$ & Čvančarová et al. (2015) \\
\hline & Ciprofloxacin & Pleurotus ostreatus & 10 & 60 (14 days) & Lac & Čvančarová et al. (2015) \\
\hline \multirow[t]{11}{*}{ Tetracyclines } & Oxytetracycline & Fusarium verticillioides KS248 & 1.5 & 34.63 (7 days) & - & Wang et al. (2018b) \\
\hline & Oxytetracycline & Penicillium janthinellum KS272 & 1.5 & 40.29 (7 days) & - & Wang et al. (2018b) \\
\hline & Oxytetracycline & T. harzianum & 250 & 92 (21 days) & - & $\begin{array}{l}\text { Ahumada-Rudolph et al. } \\
\text { (2016) }\end{array}$ \\
\hline & Oxytetracycline & T. deliquescens & 250 & 85 (21 days) & - & $\begin{array}{l}\text { Ahumada-Rudolph et al. } \\
\text { (2016) }\end{array}$ \\
\hline & Oxytetracycline & P. crustosum & 250 & 83 (21 days) & - & $\begin{array}{l}\text { Ahumada-Rudolph et al. } \\
\text { (2016) }\end{array}$ \\
\hline & Tetracycline & Phanerochaete chrysosporium & 10 & 80 (3 days) & Lip, mnp & Qing et al. (2018) \\
\hline & Tetracycline & Trichoderma harzianum & 250 & 92 (21 days) & - & $\begin{array}{l}\text { Ahumada-Rudolph et al. } \\
\text { (2016) }\end{array}$ \\
\hline & Tetracycline & Trichoderma deliquescens & 250 & 85 (21 days) & - & $\begin{array}{l}\text { Ahumada-Rudolph et al. } \\
\text { (2016) }\end{array}$ \\
\hline & Tetracycline & Penicillium crustosum & 250 & 83 (21 days) & - & $\begin{array}{l}\text { Ahumada-Rudolph et al. } \\
\text { (2016) }\end{array}$ \\
\hline & Tetracycline & Rhodotorula mucilaginosa & 250 & 73 (21 days) & -- & $\begin{array}{l}\text { Ahumada-Rudolph et al. } \\
\text { (2016) }\end{array}$ \\
\hline & Tetracycline & Talaromyces atroroseus & 250 & 72 (21 days) & - & $\begin{array}{l}\text { Ahumada-Rudolph et al. } \\
\text { (2016) }\end{array}$ \\
\hline \multirow[t]{5}{*}{ Beta-lactam } & Oxacillin & Leptosphaerulina sp & 16 & 100 (6 days) & Lac and mnp and lip & Copete-Pertuz et al. (2018) \\
\hline & Cloxacillin & Leptosphaerulina sp & 17.5 & 100 (6 days) & Lac and mnp and lip & Copete-Pertuz et al. (2018) \\
\hline & Dicloxacillin & Leptosphaerulina sp & 19 & 100 (6 days) & Lac and mnp and lip & Copete-Pertuz et al. (2018) \\
\hline & Cephadroxyl & $\begin{array}{l}\text { Leptosphaerulina sp. } \\
\text { CECT20913 }\end{array}$ & 15.2 & 100 (15 days) & Lac and mnp & Pérez Grisales et al. (2019) \\
\hline & Cephadroxyl & $\begin{array}{l}\text { Trametes versicolor ATCC } \\
42530\end{array}$ & 6 & 100 (15 days) & Lac and mnp & Pérez Grisales et al. (2019) \\
\hline
\end{tabular}

Notes: Lac: laccase; LiP: lignin peroxidase; MnP: manganese peroxidase; MIP: manganese-independent peroxidase; CGMCC: China General Microbiological Culture Collection Center.

\section{Physical Adsorption}

Physical adsorption is the adsorption of antibiotic molecules on the adsorbent through intermolecular forces. Commonly used adsorbents include activated carbon, modified activated carbon, and other molecular sieve pore structure substances. Ahmed and Theydan (2014) used microwave technology to prepare activated carbon and had high adsorption rates for both ciprofloxacin (CIP) and norfloxacin. Choi et al. (2008) successfully used granular activated carbon. In a different study, Chen and
Huang (2010) analyzed the strong adsorption of alumina to three tetracycline antibiotics (chlortetracycline, oxytetracycline, and tetracycline). The efficiency of an adsorbent is related to the $\mathrm{pH}$ of the solution. Adsorbents are widely used in wastewater management because they not only adsorb small molecules, such as antibiotics, but also some heavy metal ions and toxic substances such as dyes. Physical adsorption is a low cost method characterized by simple preparation of the adsorbent, no high technical requirements, simple operation, large specific 
TABLE 10 | Antibiotic enzyme systems.

\begin{tabular}{|c|c|c|c|c|c|c|c|}
\hline Antibiotics & & Enzyme & pH & $\begin{array}{l}\text { Temperature } \\
\text { ('C) }\end{array}$ & Time & $\begin{array}{c}\text { Removal } \\
\text { rate } \\
(\%)\end{array}$ & References \\
\hline \multirow[t]{12}{*}{ Sulfonamides } & Sulfamethoxazole & Laccase-syringaldehyde & 6 & 25 & $<1 \mathrm{~h}$ & 100 & $\begin{array}{l}\text { Kumar et al. } \\
(2005)\end{array}$ \\
\hline & Sulfamethoxazole & Laccase-acetosyringone & 6 & 25 & $<1 \mathrm{~h}$ & 100 & $\begin{array}{l}\text { Kumar et al. } \\
(2005)\end{array}$ \\
\hline & Sulfonamide* & Laccase-syringaldehyde & 6 & 25 & $180 \mathrm{~min}$ & $>97$ & Ding et al. (2016) \\
\hline & Sulfadimethoxine & $\begin{array}{l}\text { Laccase-2-2'-azino-bis-(3-ethylbenzothiazoline-6- } \\
\text { sulfonic acid) diammonium salt }\end{array}$ & 4.1 & 30 & $30 \mathrm{~min}$ & 85 & $\begin{array}{l}\text { Weng et al. } \\
(2011)\end{array}$ \\
\hline & Sulfadimethoxine & Laccase-violuric acid & 4.1 & 30 & $30 \min$ & 81 & $\begin{array}{l}\text { Weng et al. } \\
(2011)\end{array}$ \\
\hline & Sulfamonomethoxine & $\begin{array}{l}\text { Laccase-2-2'-azino-bis-(3-ethylbenzothiazoline-7- } \\
\text { sulfonic acid) diammonium salt }\end{array}$ & 4.1 & 30 & $30 \min$ & 67 & $\begin{array}{l}\text { Weng et al. } \\
(2011)\end{array}$ \\
\hline & Sulfamonomethoxine & Laccase-violuric acid & 4.1 & 30 & $30 \min$ & 82 & $\begin{array}{l}\text { Weng et al. } \\
(2011)\end{array}$ \\
\hline & Sulfadimethoxine & Laccase-4-hydroxybenzyl alcohol & 4.1 & 30 & $330 \mathrm{~min}$ & 67 & $\begin{array}{l}\text { Weng et al. } \\
(2011)\end{array}$ \\
\hline & Sulfadimethoxine & Laccase-syringaldehyde & 4.1 & 30 & $330 \mathrm{~min}$ & $89-90$ & $\begin{array}{l}\text { Weng et al. } \\
\text { (2011) }\end{array}$ \\
\hline & Sulfamonomethoxine & Laccase-5-hydroxybenzyl alcohol & 4.1 & 30 & $330 \mathrm{~min}$ & 70 & $\begin{array}{l}\text { Weng et al. } \\
\text { (2011) }\end{array}$ \\
\hline & Sulfamonomethoxine & Laccase-syringaldehyde & 4.1 & 30 & $330 \mathrm{~min}$ & 83 & $\begin{array}{l}\text { Weng et al. } \\
(2011)\end{array}$ \\
\hline & Sulfamethoxazole & Laccase & 4.5 & 30 & $24 \mathrm{~h}$ & 50 & Guo et al. (2014) \\
\hline \multirow[t]{3}{*}{ Fluoroquinolones } & Norfloxacin & Chloroperoxidase (CPO) $-\mathrm{H}_{2} \mathrm{O}_{2}$ & 5 & 25 & $25 \min$ & 82.18 & Zhao et al. (2017) \\
\hline & Ciprofloxacin & $\begin{array}{l}\text { Combi-CLEA (laccase and versatile peroxidase and } \\
\text { glucose oxidase) }\end{array}$ & 3 & 30 & $72 \mathrm{~h}$ & $80 / 90$ & $\begin{array}{l}\text { Touahar et al. } \\
(2014)\end{array}$ \\
\hline & Fluoroquinolone* & Laccase-syringaldehyde + soil & 6 & 25 & $180 \mathrm{~min}$ & $>95$ & Ding et al. (2016) \\
\hline \multirow[t]{5}{*}{ Tetracyclines } & Trimethoprim & $\begin{array}{l}\text { Combi-CLEA (laccase and versatile peroxidase and } \\
\text { glucose oxidase) }\end{array}$ & 3 & 30 & $72 \mathrm{~h}$ & $50-70$ & $\begin{array}{l}\text { Touahar et al. } \\
(2014)\end{array}$ \\
\hline & Tetracycline* & Laccase-syringaldehyde + soil & 6 & 25 & $180 \mathrm{~min}$ & $>95$ & Ding et al. (2016) \\
\hline & Tetracycline & Laccase & 7 & 20 & $18 \mathrm{~h}$ & 78 & $\begin{array}{l}\text { Llorca et al. } \\
(2014)\end{array}$ \\
\hline & Tetracycline & Crude lignin peroxidase-veratryl alcohol & 4.2 & 37 & $30 \mathrm{~min}$ & 99 & Wen et al. (2009) \\
\hline & Tetracycline & Crude lignin peroxidase & 3 & 37 & $30 \mathrm{~min}$ & 92 & $\begin{array}{l}\text { Xingxing et al., } \\
\text { (2016) }\end{array}$ \\
\hline Macrolides & Erythromycin & EreB esterase & 7 & 37 & $16 \mathrm{~h}$ & 52 & $\begin{array}{l}\text { Llorca et al. } \\
(2014)\end{array}$ \\
\hline
\end{tabular}

surface area, and strong capacity for antibiotic adsorption. However, due to weak intermolecular interaction, the adsorbed antibiotics can easily escape under the influence of the external environment and cause secondary pollution, and is thus limited in its use to cases with low antibiotic concentrations.

\section{Membrane Filtration}

Membrane separation technology uses micro- and nano-porous membranes to intercept or reverse osmosis of antibiotics in water for purification purposes. In practice, membrane separation is commonly used in conjunction with other methods to remove antibiotics from the aquatic environment. Wang et al. (2017a) studied the removal efficiency and influencing factors of tetracycline in water using a magnetic flocculation-membrane separation technique. Yang et al. (2020) made membrane bioreactors (MBRs) more effective for antibiotic treatment by adding rice straw to improve denitrification. In a study by Pérez and Barceló (2008), a laboratory-scale membrane bioreactor achieved a $56 \%$ elimination of diclofenac metabolite $4^{\prime}$ hydroxydiclofenac. The use of membrane filtration is a physical process that does not add any chemical reagents, is green, and has good selective filtration. However, because of the small size of the antibiotic molecule and the tendency of other contaminants to clog the pore size, the membrane module needs to be replaced frequently, which is costly.

\section{Degradation of Antibiotics by Photolysis}

The separation of electrons and holes generated by semiconductor photocatalysts under light excitation leads to the generation of a large number of oxygen radicals in aquatic environments, thus oxidizing any present antibiotics. Titanium oxide has a high degradation rate for antibiotics in water, however, owing to the narrow spectral absorption of pure $\mathrm{TiO}_{2}$, researchers often modify it to efficiently degrade antibiotics in wastewater. Mushtaq et al. (2020) used titanium isopropoxide as a titanium precursor to synthesize peptide-based nanoparticles to study the degradation of NOR. Mountassir et al. (2020) constructed recyclable $\mathrm{LDH}_{-} \mathrm{TiO}_{2}$ nanocomposites to degrade sulfamethyisoxazole under UV radiation, where $\mathrm{TiO}_{2}$ can be reused, to reduce the threat to 
water resources. Furthermore, researchers are developing new photoelectric systems and explore the use of other metal mineral salts as catalysts to degrade antibiotics. Chang et al. (2017a) constructed a new photoelectric catalytic (PEC) coupled electroenzyme-catalyzed (EEC) oxidation system that degraded up to $98.7 \%$ chloramphenicol within $10 \mathrm{~h}$. Eswar et al. (2017) investigated the performance of reticulated $\mathrm{CuO}$ photocatalytic degradation of tetracycline in water. Cao et al. (2018) loaded $\mathrm{AgPO}_{4}$-modified $\mathrm{BiVO}_{4}$ on a photoanode on conductive glass, which effectively degraded levofloxacin in water. Photodegradation, a widely used method for the degradation of antibiotics, is green and environmentally friendly, relying on water molecules to provide hydroxyl radicals and oxygen radicals to degrade antibiotics, thus avoiding the possibility of secondary pollution. However, because of the high construction cost of photoelectrodes, it can only be used short-range, which comes with certain limitations. At the same time, some suspended solids and deep pigments in the wastewater obstruct the passage of light and negatively affect the photocatalytic effect.

\section{Chemical Oxidative Degradation of Antibiotics}

Chemical oxidation is the degradation of antibiotics through free radicals produced by a chemical reaction that react directly with the antibiotic, causing its chemical bonds to break or decompose. However, this type of method produces a large amount of secondary pollution.

Ji et al. (2015), Yan et al. (2015) used thermal activation of peroxynitrite to produce sulfate radicals to effectively degrade sulfonamide antibiotics, but the effect is not very stable. Gaffney et al. (2015), Nassar et al. (2018) found that chlorination and oxidation selectively removed sulfonamide antibiotics from water, but the degradation effect was greatly influenced by the concentration of the antibiotics and $\mathrm{pH}$.

Compared with earlier oxidative degradation by strong oxidants, Fenton oxidation has greatly reduced the chemical pollution of the environment (Tunç et al., 2012; Tunç et al., 2013; Le et al., 2016; Weng et al., 2020). It can effectively oxidize and remove the difficult-to-degrade organic substances that cannot be removed by traditional wastewater treatment technology. Ioannou-Ttofa et al. (2018) used light illumination combined with the Fenton oxidation technique to degrade ampicillin in water, and showed that the $\mathrm{pH}$ had a great influence on the degradation result. The degradation rate of ampicillin increased with an increase in solution $\mathrm{pH}$ under acidic conditions.

\section{Biodegradation of Antibiotics}

Biodegradation of antibiotics is the use of microorganisms, microbes, and enzymes to break down antibiotics in the environment. It generally does not cause secondary pollution, can be used in a variety of environments, and is an environmentally friendly disposal method.

\section{Plant Adsorption}

Plants take up antibiotics from river water and bottom sediments through roots, stems, and leaves, and then transport them through transpiration or degradation by microorganisms enriched by the roots (Susarla et al., 2002), thus reducing the content of pollutants in the aqueous environment. Plant removal of antibiotics is currently performed mainly by the construction of plant floating beds, artificial wetlands, and other environmental management technologies. Because of their low cost and ease of operation, artificial floating beds are more common than artificial wetlands (Figure 6), even though artificial wetlands can simultaneously deal with a variety of environmental pollution problems and have self-healing functions that can be used for long periods. As shown in Table 7, the type of antibiotic that can be removed from the water column and the antibiotic removal efficiency vary for different plants.

\section{Degradation of Antibiotics Using Activated Sludge}

Activated sludge is a collective term for communities of microorganisms and the organic and inorganic materials to which they are attached, and is used for biosorption of antibiotics, biodegradation of antibiotics, and flocculation. The complex microorganisms in the activated sludge form a complex food chain with organic nutrients in the wastewater, and the degradation of antibiotics is achieved through the action of the microbial community. Composting with activated sludge removes contaminants through adsorption and microbial biodegradation. Studies have shown (Zhang et al., 2019b; Zhu et al., 2020) that antibiotics can be removed either by microbial nitrification (autotrophic biodegradation) or by microbial chemical oxygen demand (COD) degradation (heterotrophic biodegradation) in activated sludge.

Terzic et al. (2018) found that activated sludge from a municipal wastewater treatment plant was able to degrade three major macrolides (erythromycin, clarithromycin, and azithromycin) and evaluated their toxicity. Their results showed that the harmful effects of the treated effluent were greatly reduced. Radjenovic et al. (2009) found that charged activated sludge influenced the adsorption of environmental quinolone antibiotics. However, while the adsorption was improved, the effect was not stable. Activated sludge for the degradation of antibiotics is generally derived from biopharmaceutical or hospital wastewater and significantly reduces post-degradation toxicity after activated sludge treatment. However, this method is highly dependent on environmental factors such as $\mathrm{pH}$, temperature, dissolved oxygen, nutrients, and toxic substances, as well as the composition and proportion of the microorganisms, which influence the degradation time. Under low dissolved oxygen conditions, irritating gases such as ammonia or sulfur dioxide are easily produced, and the proportion of nutrients in the wastewater needs to be adjusted frequently; otherwise, the degradation efficiency is low.

The use of activated sludge to decompose antibiotics could have some disadvantages. For example, bacterial fermentation in activated sludge may occur to complete the decomposition of antibiotics. The exposure of bacteria to antibiotics may then lead to the production and proliferation of ARGs, resulting in the emergence of novel types of genetic pollutants (Zeng et al., 2019) 


\section{Degradation by Microbial Strains}

As a result of prolonged exposure to antibiotics, strains of bacteria become resistant to antibiotics and may even break them down. The biodegradation of antibiotics is dominated by microbial decomposition. However, the antibiotic degradation ability of strains is influenced by many factors such as the antibiotic species, strain type, carbon source, nitrogen source, temperature, and wastewater components (heavy metal ions, COD, etc., ). In recent years, many bacterial strains with antibiotic degradation abilities have been isolated through screening, enrichment, and domestication, and mainly degrade sulfonamides and tetracyclines. This is most likely due to the fact that these two types of antibiotics are more likely to be adsorbed onto sediment and thus remain in a stationary environment for longer periods (Mulla et al., 2018). Table 8 lists the antibioticdegrading bacterial strains reported in the literature over the last three years.

The microorganisms that degrade antibiotics can be divided into single and mixed strains, some of which are listed in Martins et al. (2018) demonstrated for the first time that sulfate-reducing flora could remove CIP, and Cordova-Kreylos and Scow (2007) observed that exposing anaerobic sediments to the antibiotic CIP sulfate-reducing bacteria (SRB) is advantageous. In addition, there have been reports that bacteria can degrade antibiotics under sulfate-reducing conditions (Jia et al., 2017) and that nitrate reduction has been used successfully in microbial remediation, where denitrifying bacteria can effectively degrade enrofloxacin and ceftiofur (Alexandrino et al., 2017) as well as CIP. Antibiotic-degrading flora are also present in many estuarine sediments enriched in antibiotics; Chang and Ren (2015) isolated tetracycline antibiotic-degrading flora in sediments of the Eren River estuary, and Harrabi et al. (2018) enriched the flora of the Douro River estuary and achieved a greater than $95 \%$ degradation for oxytetracycline and enrofloxacin.

Fungi are more tolerant to high concentrations of pollutants than bacteria and, therefore, are more advantageous in the degradation of antibiotics. Numerous studies have shown that it is feasible to use fungi to degrade antibiotics present in the environment. In Table $\mathbf{9}$, the antibiotic-degrading fungi reported in the literature during the last 5 years are listed.

\section{Enzymatic Degradation}

Microorganisms can produce enzymes that degrade antibiotics, such as $\beta$-lactamases, which can cleave the $\beta$-lactam rings of cyanotoxins and cephalosporins. Based on the substrate specificity of $\beta$-lactamases, they can be roughly divided into three categories: penicillinases, cephalosporinases, and oximetype cephalosporinases. Penicillin enzymes easily decompose penicillin antibiotics, while cephalosporin enzymes have a higher activity in decomposing cephalosporin antibiotics, and the oxime cephalosporin enzymes have a decomposing effect on both penicillin and cephalosporin, but are especially good at decomposing oxime cephalosporin. To efficiently degrade antibiotics, enzyme systems can be constructed. Table 10 lists the enzyme systems that have been used to degrade antibiotics in recent years.
The construction of various enzyme systems and the use of immobilized enzymes to degrade antibiotics, considered a breakthrough in the field of environmental management, was motivated by the fact that enzymes are easily inactivated in the environment and cannot be used in large quantities in practical applications. Gao et al. (2017b) using magnetic nanoparticles $\mathrm{Fe}_{3} \mathrm{O}_{4}$ to immobilize $\beta$-lactamase to degrade penicillin, the efficiency remained above $95 \%$ after 35 times of repeated use; Zhang et al. (2020a) used in situ immobilized laccase to degrade tetracycline and ampicillin and achieved a degradation efficiency in water close to $100 \%$. Simón-Herrero et al. (2019) found that laccase immobilized on polyimide erogels used to remove carbamazepine yielded a degradation efficiency of $74 \%$. In a different study, Zdarta et al. (2019) successfully degraded tetracycline by laccase immobilized with electrospun materials using 1-hydroxybenzotriazole as a medium. For degradation experiments, Becker et al. (2017) constructed an enzyme membrane reactor using a mixture of immobilized laccase and eugenol for the degradation of 38 antibiotics. Their results showed that after $24 \mathrm{~h}$ the reactor degraded 32 types of antibiotics with a degradation rate of greater than $50 \%$.

At present, the existing enzymes are mainly $\beta$-lactamase types for the degradation of lactam antibiotics, laccase, and other strong oxidative enzymes for the nonselective oxidative degradation of antibiotics. Due to the rapid development of immobilized materials, an increasing number of enzymes are immobilized and are already used in actual sites to manage antibiotics pollution (Shao et al., 2019; Liang and Hu, 2020; Shakerian et al., 2020; Zhang et al., 2020a), resulting in an improvement of the stability of the enzymes and the recycling and reuse of them, thus reducing the cost. Over the past two years, immobilized lacquer enzymes have been applied to the degradation of antibiotics, and some other strongly oxidizing enzymes have gradually entered the view of the general researcher and are applied to oxidative degradation of antibiotics by immobilization.

\section{SUMMARY AND OUTLOOK}

Antibiotics have greatly polluted the environment globally. Among the prevalent antibiotic pollution treatments, physical adsorption cannot degrade antibiotics, chemical oxidation is likely to cause secondary pollution, photodegradation is expensive. Biodegradation of antibiotics, however, is attracting increasing attention because of its low cost, easy operation, and lack of secondary pollution. Nevertheless, both microbial degradation and activated sludge degradation will inevitably lead to the proliferation of ARGs. Therefore, using purified antibiotic-degrading enzymes for the degradation of antibiotics poses a good alternative. Synthesizing related enzymes in vitro or constructing engineered bacteria to produce enzymes would reduce the cost of this approach, making it even more attractive. Therefore, enzyme degradation is becoming the future mainstream of environmental management. The main remaining problem is whether the antibiotic degradation products have toxicity. Although the degradation products of 
antibiotics are tested for bacterial toxicity, whether there may be a long-term toxicity problem has yet to be determined. However, it is undeniable that the toxicity of enzymatic antibiotics will be greatly reduced. Once the toxicity problem is solved, antibioticdegrading enzymes may be used in a variety of wastewater treatments.

Antibiotics in the environment can be enriched in humans through the food chain, and they can be very harmful to young children and pregnant women. In addition, antibiotic contamination increases the development of superbugs. However, there is a lack of mandatory standards for the limits of antibiotic fugitive levels in the surface water. Thus, there is an urgent need to control antibiotics in water bodies. China's vast territory has a diverse climate, a diverse industrial layout, and unbalanced economic development. All the factors above influence the distribution of antibiotics in China, and these factors make it challenging for governments to control antibiotics. In recent years, China has attached great importance to the issue of antibiotic contamination, and the government has made significant improvements in antibiotic stewardship; however, there are still some shortcomings, such as, the antibiotic regulatory system and antibiotic managementrelated standards are inadequate to effectively combat antibiotics

\section{REFERENCES}

Ahmed, M. J., and Theydan, S. K. (2014). Fluoroquinolones Antibiotics Adsorption onto Microporous Activated Carbon from Lignocellulosic Biomass by Microwave Pyrolysis. J. Taiwan Inst. Chem. Eng. 45, 219-226. doi:10.1016/ j.jtice.2013.05.014

Ahumada-Rudolph, R., Novoa, V., Sáez, K., Martínez, M., Rudolph, A., TorresDiaz, C., et al. (2016). Marine Fungi Isolated from Chilean Fjord Sediments Can Degrade Oxytetracycline. Environ. Monit. Assess. 188. doi:10.1007/s10661-0165475-0

Alexandrino, D. A. M., Mucha, A. P., Almeida, C. M. R., Gao, W., Jia, Z., and Carvalho, M. F. (2017). Biodegradation of the Veterinary Antibiotics Enrofloxacin and Ceftiofur and Associated Microbial Community Dynamics. Sci. Total Environ. 581-582, 359-368. doi:10.1016/ j.scitotenv.2016.12.141

Aydin, S. (2016). Enhanced Biodegradation of Antibiotic Combinations via the Sequential Treatment of the Sludge Resulting from Pharmaceutical Wastewater Treatment Using White-rot Fungi Trametes versicolor and Bjerkandera Adusta. Appl. Microbiol. Biotechnol. 100, 6491-6499. doi:10.1007/s00253-016-7473-0

Bao, Y., Li, F. W., and Wen, D. H. (2021). Antibiotic Contamination in Mariculture in China. Mar. Environ. Sci. 40, 294-302.

Baran, W., Adamek, E., Ziemiańska, J., and Sobczak, A. (2011). Effects of the Presence of Sulfonamides in the Environment and Their Influence on Human Health. J. Hazard. Mater. 196, 1-15. doi:10.1016/j.jhazmat.2011.08.082

Becker, D., Rodríguez-Mozaz, S., Insa, S., Schoevaart, R., Barceló, D., de Cazes, M., et al. (2017). Removal of Endocrine Disrupting Chemicals in Wastewater by Enzymatic Treatment with Fungal Laccases. Org. Process. Res. Dev. 21, 480-491. doi:10.1021/acs.oprd.6b00361

Ben, W., Pan, X., and Qiang, Z. (2013). Occurrence and Partition of Antibiotics in the Liquid and Solid Phases of Swine Wastewater from Concentrated Animal Feeding Operations in Shandong Province, China. Environ. Sci. Process. Impacts 15, 870-875. doi:10.1039/c3em30845f

Bengtsson-Palme, J. (2017). Antibiotic Resistance in the Food Supply Chain: where Can Sequencing and Metagenomics Aid Risk Assessment? Curr. Opin. Food Sci. 14, 66-71. doi:10.1016/j.cofs.2017.01.010 pollution. In addition, factory emissions do not meet safety standards. Furthermore, there are no institutions specializing in antibiotic use and management to monitor the use of antibiotics and subsequent pollution management. Finally, scientific guidance on drug use is still required to discourage and prevent antibiotic abuse.

\section{AUTHOR CONTRIBUTIONS}

WT and LM conceived the idea of the review, LZ collected literature, CL and LT wrote the manuscript. All authors contributed to the article.

\section{FUNDING}

This work was supported by the Beijing Collaborative innovation center for eco-environmental improvement with forestry and fruit trees, Beijing University of Agriculture; Beijing Natural Science Foundation (Grant No. 7174283). Beijing Advanced Innovation Center for Tree Breeding by Molecular Design, Beijing University of Agriculture.

Bilal, M., Ashraf, S. S., Barceló, D., and Iqbal, H. M. N. (2019). Biocatalytic Degradation/redefining "Removal" Fate of Pharmaceutically Active Compounds and Antibiotics in the Aquatic Environment. Sci. Total Environ. 691, 1190-1211. doi:10.1016/j.scitotenv.2019.07.224

Bitchava, K., and Nengas, I. (2010). Antibacterial Drugs in Products Originating from Aquaculture: Assessing the Risks to Public Welfare. Medit. Mar. Sci. 11, 33-41. doi:10.12681/mms.89

Bo, D. (2017). Study on Photocatalytic Degradation of Antibiotic Wastewater. Management \& Technology of SME, 138-140. doi:10.3969/j.issn.16731069.2017.15.067

Brain, R. A., Hanson, M. L., Solomon, K. R., and Brooks, B. W. (2008). Aquatic Plants Exposed to Pharmaceuticals: Effects and Risks. Rev. Environ. Contam. T. 192, 67-115. doi:10.1007/978-0-387-71724-1_3

Brain, R. A., Johnson, D. J., Richards, S. M., Sanderson, H., Sibley, P. K., and Solomon, K. R. (2004). Effects of 25 Pharmaceutical Compounds to Lemna Gibba Using a Seven-Day Static-Renewal Test. Environ. Toxicol. Chem. 23, 371-382. doi:10.1897/02-576

Briones, R. M., Sarmah, A. K., and Padhye, L. P. (2016). A Global Perspective on the Use, Occurrence, Fate and Effects of Anti-diabetic Drug Metformin in Natural and Engineered Ecosystems. Environ. Pollut. 219, 1007-1020. doi:10.1016/ j.envpol.2016.07.040

Cao, D., Wang, Y., Qiao, M., and Zhao, x. (2018). Enhanced Photoelectrocatalytic Degradation of Norfloxacin by an Ag 3 PO 4/BiVO 4 Electrode with Low Bias. J. Catal. 360, 240-249. doi:10.1016/j.jcat.2018.01.017

Cao, J., Liu, F., Liu, S., Wang, J., Zhu, B., Shi, Y., et al. (2021). Identification of Antibiotic Resistance Genes and Associated mobile Genetic Elements in Permafrost. Sci. China Life Sci. 1-4.

Chang, B.-V., and Ren, Y.-L. (2015). Biodegradation of Three Tetracyclines in River Sediment. Ecol. Eng. 75, 272-277. doi:10.1016/j.ecoleng.2014.11.039

Chen, H., Liu, S., Xu, X.-R., Diao, Z.-H., Sun, K.-F., Hao, Q.-W., et al. (2018). Tissue Distribution, Bioaccumulation Characteristics and Health Risk of Antibiotics in Cultured Fish from a Typical Aquaculture Area. J. Hazard. Mater. 343, 140-148. doi:10.1016/j.jhazmat.2017.09.017

Chen, H., Liu, S., Xu, X.-R., Liu, S.-S., Zhou, G.-J., Sun, K.-F., et al. (2015). Antibiotics in Typical marine Aquaculture Farms Surrounding Hailing Island, South China: Occurrence, Bioaccumulation and Human Dietary Exposure. Mar. Pollut. Bull. 90, 181-187. doi:10.1016/j.marpolbul.2014.10.053 
Chen, J., Ying, G. G., Wei, X. D., Liu, Y. S., Liu, S. S., Hu, L. X., et al. (2016). Removal of Antibiotics and Antibiotic Resistance Genes from Domestic Sewage by Constructed Wetlands: Effect of Flow Configuration and Plant Species. Sci. Total Environ. 571, 974-982. doi:10.1016/j.scitotenv.2016.07.085

Chen, J., Tong, T., Jiang, X., and Xie, S. (2020a). Biodegradation of Sulfonamides in Both Oxic and Anoxic Zones of Vertical Flow Constructed Wetland and the Potential Degraders. Environ. Pollut. 265, 115040. doi:10.1016/ j.envpol.2020.115040

Chen, L., Li, H., Liu, Y., Cui, Y., Li, Y., and Yang, Z. (2019). Distribution, Residue Level, Sources, and Phase Partition of Antibiotics in Surface Sediments from the Inland River: a Case Study of the Xiangjiang River, South-central China. Environ. Sci. Pollut. Res. 27, 2273-2286. doi:10.1007/s11356-019-06833-0

Chen, Q., Guo, X., Hua, G., Li, G., Feng, R., and Liu, X. (2017). Migration and Degradation of Swine Farm Tetracyclines at the River Catchment Scale: Can the Multi-Pond System Mitigate Pollution Risk to Receiving Rivers?. Environ. Pollut. 220, 1301-1310. doi:10.1016/j.envpol.2016.11.004

Chen, W.-R., and Huang, C.-H. (2010). Adsorption and Transformation of Tetracycline Antibiotics with Aluminum Oxide. Chemosphere 79, 779-785. doi:10.1016/j.chemosphere.2010.03.020

Chen, W. P., Peng, C. W., Yang, Y., and Wu, Y. M. (2017b). [Distribution Characteristics and Risk Analysis of Antibiotic in the Groundwater in Beijing]. Huan Jing Ke Xue 38, 5074-5080. doi:10.13227/j.hjkx.201704287

Chen, Y., Cui, K., Huang, Q., Guo, Z., Huang, Y., Yu, K., et al. (2020b). Comprehensive Insights into the Occurrence, Distribution, Risk Assessment and Indicator Screening of Antibiotics in a Large Drinking Reservoir System. Sci. Total Environ. 716, 137060. doi:10.1016/j.scitotenv.2020.137060

Chen, Y., Zhou, J. L., Cheng, L., Zheng, Y. Y., and Xu, J. (2017a). Sediment and Salinity Effects on the Bioaccumulation of Sulfamethoxazole in Zebrafish (Danio rerio). Chemosphere 180, 467-475. doi:10.1016/ j.chemosphere.2017.04.055

Cheng, D., Liu, X., Wang, L., Gong, W., Liu, G., Fu, W., et al. (2014). Seasonal Variation and Sediment-Water Exchange of Antibiotics in a Shallower Large lake in North China. Sci. Total Environ. 476-477, 266-275. doi:10.1016/ j.scitotenv.2014.01.010

Cheng, J., Du, H., Zhang, T., and Wang, W. (2017b). Isolation and Identification of Tetracyclines Degrading Bacteria. J. Nucl. Agric. Sci. 31, 884-888. doi:10.11869/ j.issn.100-8551.2017.05.0884

Cheng, L., Liu, L., Yan, K., and Zhang, J. (2017a). Visible Light-Driven Photoelectrocatalysis Coupling with Electroenzymatic Process for Degradation of Chloramphenicol. Chem. Eng. J. 330, 1380-1389. doi:10.1016/j.cej.2017.07.170

Choi, K.-J., Kim, S.-G., and Kim, S.-H. (2008). Removal of Antibiotics by Coagulation and Granular Activated Carbon Filtration. J. Hazard. Mater. 151, 38-43. doi:10.1016/j.jhazmat.2007.05.059

Chuan, R., Yanan, S., Yinghui, W., Yuanyuan, Z., and Kefu, Y. (2018). Formation of Disinfection Byproducts from Sulfamethoxazole during Sodium Hypochlorite Disinfection of marine Culture Water. Environ. Sci. Pollut. Res. International. 25, 33196-33206. doi:10.1007/s11356-018-3278-2

Conkle, J. L., Lattao, C., White, J. R., and Cook, R. L. (2010). Competitive Sorption and Desorption Behavior for Three Fluoroquinolone Antibiotics in a Wastewater Treatment Wetland Soil. Chemosphere 80, 1353-1359. doi:10.1016/j.chemosphere.2010.06.012

Copete-Pertuz, L. S., Plácido, J., Serna-Galvis, E. A., Torres-Palma, R. A., and Mora, A. (2018). Elimination of Isoxazolyl-Penicillins Antibiotics in Waters by the Ligninolytic Native Colombian Strain Leptosphaerulina Sp. Considerations on Biodegradation Process and Antimicrobial Activity Removal. Sci. Total Environ. 630, 1195-1204. doi:10.1016/j.scitotenv.2018.02.244

Córdova-Kreylos, A. L., and Scow, K. M. (2007). Effects of Ciprofloxacin on Salt Marsh Sediment Microbial Communities. ISME J. 1, 585-595. doi:10.1038/ ismej.2007.71

Čvančarová, M., Filipová, A., and Cajthaml, T. (2015). Biotransformation of Fluoroquinolone Antibiotics by Ligninolytic Fungi - Metabolites, Enzymes and Residual Antibacterial Activity. Chemosphere 136. doi:10.1016/ j.chemosphere.2014.12.012

Dai, H. (2019). Analysis of Antibiotic Pollution Characteristics and Ecological Risk Assessment in Water Sources of Qingyuan City and Dongguan City. Xi'an Polytechnic University. doi:10.1007/978-3-030-02874-9
Dan, A., Dai, Y. N., Chen, C. X., Wang, S. Y., and Tao, R. (2013). Removal and Factors Influencing Removal of Sulfonamides and Trimethoprim from Domestic Sewage in Constructed Wetlands. Bioresour. Technol. 146, 363-370. doi:10.1016/j.biortech.2013.07.050

Dethlefsen, L., McFall-Ngai, M., Relman, D. A., Dethlefsen, L., and McFallNgai, M. (2007). An Ecological and Evolutionary Perspective on HumanMicrobe Mutualism and Disease. Nature 449, 811-818. doi:10.1038/ nature 06245

Di, X., Cui, X., Sun, K., Zhang, K., and Huang, T. (2019). Determination of Antibiotics Residues in Drinking Water Sample by Solid-phase Extraction/high Performance Liquid Chromatography Tandem Mass Spectrometry. Mod. Chem. Ind. 39, 243-247. doi:10.16606/j.cnki.issn0253-4320.2019.12.051

Dik, V. K., Van Oijen, M. G. H., Smeets, H. M., and Siersema, P. D. (2016). Frequent Use of Antibiotics Is Associated with Colorectal Cancer Risk: Results of a Nested Case-Control Study. Dig. Dis. Sci. 61, 255-264. doi:10.1007/s10620015-3828-0

Ding, H. (2018). Study on the Characteristics of Antibiotics in Poyang Lake and the Adsorption and Degradation of Typical Antibiotics. Wuhan, China: Wuhan University.

Ding, H., Wu, Y., Zhang, W., Zhong, J., Lou, Q., Yang, P., et al. (2017). Occurrence, Distribution, and Risk Assessment of Antibiotics in the Surface Water of Poyang Lake, the Largest Freshwater lake in China. Chemosphere 184, 137-147. doi:10.1016/j.chemosphere.2017.05.148

Ding, H., Wu, Y., Zou, B., Lou, Q., Zhang, W., Zhong, J., et al. (2016). Simultaneous Removal and Degradation Characteristics of Sulfonamide, Tetracycline, and Quinolone Antibiotics by Laccase-Mediated Oxidation Coupled with Soil Adsorption. J. Hazard. Mater. 307, 350-358. doi:10.1016/j.jhazmat.2015.12.062

Dong, D., Zhang, L., Liu, S., Guo, Z., and Hua, X. (2016). Antibiotics in Water and Sediments from Liao River in Jilin Province, China: Occurrence, Distribution, and Risk Assessment. Environ. Earth Sciearth Sci. 75, 1202. doi:10.1007/s12665016-6008-4

Dong, W., Xin, H., Hongbo, Z., Zhang, Y., Quan, X., and Mu, H. (2020). Research on the Pollution and Distribution Characteristics of Typical Antibiotics in Dalian Biliuhe Reservoir and River. J. Dalian Univ. Technol. 60, 119-127.

Dubreil, E., Gautier, S., Fourmond, M.-P., Bessiral, M., Gaugain, M., Verdon, E., et al. (2017). Validation Approach for a Fast and Simple Targeted Screening Method for 75 Antibiotics in Meat and Aquaculture Products Using LC-MS/ MS. Food Additives \& Contaminants: A 34, 453-468. doi:10.1080/ 19440049.2016.1230278

Dutta, J., and Mala, A. A. (2020). Removal of Antibiotic from the Water Environment by the Adsorption Technologies: a Review. Water Sci. Technol. 82, 401-426. doi:10.2166/wst.2020.335

Eswar, N. K., Singh, S. A., and Madras, G. (2018). Photoconductive Network Structured Copper Oxide for Simultaneous Photoelectrocatalytic Degradation of Antibiotic (Tetracycline) and Bacteria (E. coli). Chem. Eng. J. 332, 757-774. doi:10.1016/j.cej.2017.09.117

Fan, Y., Ji, Y., Kong, D., Lu, J., and Zhou, Q. (2015). Kinetic and Mechanistic Investigations of the Degradation of Sulfamethazine in Heat-Activated Persulfate Oxidation Process. J. Hazard. Mater. 300, 39-47. doi:10.1016/ j.jhazmat.2015.06.058

Fang, L., Wei, Q., Wang, Y., Yang, L., Li, Z., and Liu, J. (2017). Source and Distribution of Typical Antibiotics in the Upper Huangpu River, Shanghai. Environ. Pollut. Control. 39, 301-306. doi:10.15985/j.cnki.10013865.2017.03.015

Feng, L., Cheng, Y., Feng, L., Zhang, S., and Liu, Y. (2017). Distribution of Typical Antibiotics and Ecological Risk Assessment in Main Waters of Three Gorges Reservoir Area Research of Environ. Sci 30, 1031-1040. doi:10.13198/ j.issn.1001-6929.2017.02.33

Feng, L., Cheng, Y., Zhang, Y., Li, Z., Yu, Y., Feng, L., et al. (2020). Distribution and Human Health Risk Assessment of Antibiotic Residues in Large-Scale Drinking Water Sources in Chongqing Area of the Yangtze River. Environ. Res. 185, 109386. doi:10.1016/j.envres.2020.109386

Feng, M. J., Zhang, Q., Song, N. H., Pu, Y. Q., Yang, Z. B., Liu, Y. H., et al. (2019). [Occurrence Characteristics and Risk Assessment of Antibiotics in Source Water of the Nanjing Reach of the Yangtze River]. Huan Jing Ke Xue 40, 5286-5293. doi:10.13227/j.hjkx.201905139 
Fernandes, J. P., Almeida, C. M. R., Pereira, A. C., Ribeiro, I. L., Reis, I., Carvalho, P., et al. (2015). Microbial Community Dynamics Associated with Veterinary Antibiotics Removal in Constructed Wetlands Microcosms. Bioresour. Technology 182, 26-33. doi:10.1016/j.biortech.2015.01.096

Forsberg, K. J., Reyes, A., Wang, B., Selleck, E. M., Sommer, M. O. A., and Dantas, G. (2012). The Shared Antibiotic Resistome of Soil Bacteria and Human Pathogens. Science 337, 1107-1111. doi:10.1126/science.1220761

Fouhy, F., Guinane, C. M., Hussey, S., Wall, R., Ryan, C. A., Dempsey, E. M., et al. (2012). High-Throughput Sequencing Reveals the Incomplete, Short-Term Recovery of Infant Gut Microbiota Following Parenteral Antibiotic Treatment with Ampicillin and Gentamicin. Antimicrob. Agents Chemother. 56, 5811-5820. doi:10.1128/AAC.00789-12

Fu, B., Chen, L., Cai, T., Yang, Q., and Ding, D. (2017). Isolation and Characterization of Norfloxacin-Degrading Bacterium Strain NOR-36. Acta Scientiae Circumstantiae. 37, 576-584. doi:10.13671/j.hjkxxb.2016.0245

Gaffney, V. d. J., Cardoso, V. V., Benoliel, M. J., and Almeida, C. M. M. (2016). Chlorination and Oxidation of Sulfonamides by Free Chlorine: Identification and Behaviour of Reaction Products by UPLC-MS/MS. J. Environ. Manage. 166, 466-477. doi:10.1016/j.jenvman.2015.10.048

Gao, N., Liu, C.-X., Xu, Q.-M., Cheng, J.-S., and Yuan, Y.-J. (2018a). Simultaneous Removal of Ciprofloxacin, Norfloxacin, Sulfamethoxazole by Co-producing Oxidative Enzymes System of Phanerochaete Chrysosporium and Pycnoporus Sanguineus. Chemosphere 195, 146-155. doi:10.1016/ j.chemosphere.2017.12.062

Gao, X. J., Fan, X. J., Chen, X. P., and Ge, Z. Q. (2017b). Immobilized $\beta$-lactamase on Fe3O4 Magnetic Nanoparticles for Degradation of $\beta$-lactam Antibiotics in Wastewater. Int. J. Environ. Sci. Technol. 15, 2203-2212. doi:10.1007/s13762017-1596-4

Goldman, E. (2004). Antibiotic Abuse in Animal Agriculture: Exacerbating Drug Resistance in Human Pathogens. Hum. Ecol. Risk Assess. Int. J. 10, 121-134. doi:10.1080/10807030490281016

Goulet, O. (2015). Potential Role of the Intestinal Microbiota in Programming Health and Disease: Figure 1. Nutr. Rev. 73 (Suppl. 1), 32-40. doi:10.1093/ nutrit/nuv039

Grenni, P., Ancona, V., and Barra Caracciolo, A. (2018). Ecological Effects of Antibiotics on Natural Ecosystems: A Review. Microchemical J. 136, 25-39. doi:10.1016/j.microc.2017.02.006

Gujarathi, N. P., Haney, B. J., Park, H. J., Wickramasinghe, S. R., and Linden, J. C. (2005). Hairy Roots of Helianthus Annuus: A Model System to Study Phytoremediation of Tetracycline and Oxytetracycline. Biotechnol. Prog. 21, 775-780. doi:10.1021/bp0496225

Guo, R., Wang, S., Chang, S., Wang, X., Zhao, X., Yang, G., et al. (2016). Distribution Characteristics of Antibiotics in Jiaxing Drinking Water Source and Urban River. Environ. Chem. 35, 1842-1852. doi:10.7524/j.issn.02546108.2016.09.2016020401

Guo, X.-1., Zhu, Z.-w., and Li, H.-l. (2014). Biodegradation of Sulfamethoxazole by Phanerochaete Chrysosporium. J. Mol. Liquids 198, 169-172. doi:10.1016/ j.molliq.2014.06.017

Guo, X.-p., Liu, X., Niu, Z.-s., Lu, D.-p., Zhao, S., Sun, X.-1., et al. (2018). Seasonal and Spatial Distribution of Antibiotic Resistance Genes in the Sediments along the Yangtze Estuary, China. Environ. Pollut. 242, 576-584. doi:10.1016/ j.envpol.2018.06.099

Guo, X.-p., Zhao, S., Chen, Y.-r., Yang, J., Hou, L.-j., Liu, M., et al. (2020). Antibiotic Resistance Genes in Sediments of the Yangtze Estuary: From 2007 to 2019. Sci. Total Environ. 744, 140713. doi:10.1016/j.scitotenv.2020.140713

Han, Q. F., Zhao, S., Zhang, X. R., Wang, X. L., Song, C., and Wang, S. G. (2020). Distribution, Combined Pollution and Risk Assessment of Antibiotics in Typical marine Aquaculture Farms Surrounding the Yellow Sea, North China. Environ. Int. 138, 105551. doi:10.1016/j.envint.2020.105551

Hao, Q., Xu, X., Chen, H., Liu, S., Chen, J., Liu, S., et al. (2017). Residual Antibiotics in the Nansha Aquaculture Area of Guangzhou. J. Trop. Oceanogr. 36, 106-113. doi:10.11978/2016001

Harrabi, M., Alexandrino, D. A. M., Aloulou, F., Elleuch, B., Liu, B., Jia, Z., et al. (2019). Biodegradation of Oxytetracycline and Enrofloxacin by Autochthonous Microbial Communities from Estuarine Sediments. Sci. Total Environ. 648, 962-972. doi:10.1016/j.scitotenv.2018.08.193

He, S., Dong, D., Zhang, X., Sun, C., Wang, C., Hua, X., et al. (2018). Occurrence and Ecological Risk Assessment of 22 Emerging Contaminants in the Jilin
Songhua River (Northeast China). Environ. Sci. Pollut. Res. 25, 24003-24012. doi:10.1007/s11356-018-2459-3

He, X. T., Wang, Q., Nie, X. P., Yang, Y. T., and Cheng, Z. (2014). [Residues and Health Risk Assessment of Sulfonamides in Sediment and Fish from Typical marine Aquaculture Regions of Guangdong Province, China]. Huan Jing Ke Xue 35, 2728-2735.

Hijosa-Valsero, M., Fink, G., Schlüsener, M. P., Sidrach-Cardona, R., MartínVillacorta, J., Ternes, T., et al. (2011b). Removal of Antibiotics from Urban Wastewater by Constructed Wetland Optimization. Chemosphere 83, 713-719. doi:10.1016/j.chemosphere.2011.02.004

Hijosa-Valsero, M., Sidrach-Cardona, R., Martín-Villacorta, J., Cruz ValseroBlanco, M., Bayona, J. M., and Bécares, E. (2011a). Statistical Modelling of Organic Matter and Emerging Pollutants Removal in Constructed Wetlands. Bioresour. Technology 102, 4981-4988. doi:10.1016/ j.biortech.2011.01.063

Hofmann, U., and Schlosser, D. (2016). Biochemical and Physicochemical Processes Contributing to the Removal of Endocrine-Disrupting Chemicals and Pharmaceuticals by the Aquatic Ascomycete Phoma Sp. UHH 5-1-03. Appl. Microbiol. Biotechnol. 100, 2381-2399. doi:10.1007/s00253-015-7113-0

Hu, Y., Yan, X., Shen, Y., Di, M., and Wang, J. (2019). Occurrence, Behavior and Risk Assessment of Estrogens in Surface Water and Sediments from Hanjiang River, Central China. Ecotoxicology 28, 143-153. doi:10.1007/s10646-0182007-4

Hu, Y., Yang, X., Li, J., Lv, N., Liu, F., Wu, J., et al. (2016). The Bacterial Mobile Resistome Transfer Network Connecting the Animal and Human Microbiomes. Appl. Environ. Microbiol. 82, 6672-6681. doi:10.1128/aem.01802-16

Huang, X., Liu, C., Li, K., Su, J., Zhu, G., and Liu, L. (2015). Performance of Vertical Up-Flow Constructed Wetlands on Swine Wastewater Containing Tetracyclines and Tet Genes. Water Res. 70, 109-117. doi:10.1016/j.watres.2014.11.048

Huddleston, J. R. (2014). Horizontal Gene Transfer in the Human Gastrointestinal Tract: Potential Spread of Antibiotic Resistance Genes. Idr 7, 167-176. doi:10.2147/idr.s48820

Ioannou-Ttofa, L., Raj, S., Prakash, H., and Fatta-Kassinos, D. (2019). Solar PhotoFenton Oxidation for the Removal of Ampicillin, Total Cultivable and Resistant E. coli and Ecotoxicity from Secondary-Treated Wastewater Effluents. Chem. Eng. J. 355, 91-102. doi:10.1016/j.cej.2018.08.057

Ji, X., Shen, Q., Liu, F., Ma, J., Xu, G., Wang, Y., et al. (2012). Antibiotic Resistance Gene Abundances Associated with Antibiotics and Heavy Metals in Animal Manures and Agricultural Soils Adjacent to Feedlots in Shanghai; China. J. Hazard. Mater. 235-236, 178-185. doi:10.1016/j.jhazmat.2012.07.040

Ji, Y., Fan, Y., Liu, K., Kong, D., and Lu, J. (2015). Thermo Activated Persulfate Oxidation of Antibiotic Sulfamethoxazole and Structurally Related Compounds. Water Res. 87, 1-9. doi:10.1016/j.watres.2015.09.005

Jia, Y., Khanal, S. K., Zhang, H., Chen, G.-H., and Lu, H. (2017). Sulfamethoxazole Degradation in Anaerobic Sulfate-Reducing Bacteria Sludge System. Water Res. 119, 12-20. doi:10.1016/j.watres.2017.04.040

Johnson, T. A., Stedtfeld, R. D., Wang, Q., Cole, J. R., Hashsham, S. A., Looft, T., et al. (2016). Clusters of Antibiotic Resistance Genes Enriched Together Stay Together in Swine Agriculture. mBio 7, e02214-02215. doi:10.1128/ mbio.02214-15

Kara, Fox. (2019). CNN. The World's Rivers Are Contaminated with Antibiotics, New Study shows[EB/OL]. Avaailable at: https://edition.cnn.com/2019/05/27/ health/antibiotics-contaminate-worlds-rivers-intl-scli/index.html (Accessed May 27, 2019).

Kenyon, S., Pike, K., Jones, D., Brocklehurst, P., Marlow, N., Salt, A., et al. (2008). Childhood Outcomes after Prescription of Antibiotics to Pregnant Women with Spontaneous Preterm Labour: 7-year Follow-Up of the ORACLE II Trial. The Lancet 372, 1319-1327. doi:10.1016/s0140-6736(08)61203-9

Kim, S.-C., and Carlson, K. (2007). Temporal and Spatial Trends in the Occurrence of Human and Veterinary Antibiotics in Aqueous and River Sediment Matrices. Environ. Sci. Technol. 41, 50-57. doi:10.1021/es060737+

Koebnick, C., Tartof, S. Y., Sidell, M. A., Rozema, E., Chung, J., Chiu, V. Y., et al. (2019). Effect of In-Utero Antibiotic Exposure on Childhood Outcomes: Methods and Baseline Data of the Fetal Antibiotic EXposure (FAX) Cohort Study. JMIR. Res. Protoc. 8, e12065. doi:10.2196/12065

Kumar, K., C. Gupta, S. S., Chander, Y., and Singh, A. K. (2005). “Antibiotic Use in Agriculture and its Impact on the Terrestrial Environment," in Advances in Agronomy (Academic Press), 1-54. doi:10.1016/s0065-2113(05)87001-4 
Kümmerer, K. (2009). Antibiotics in the Aquatic Environment - A Review - Part I. Chemosphere 75, 417-434. doi:10.1016/j.chemosphere.2008.11.086

Kummerer, K. (2009). Antibiotics in the Aquatic Environment - A Review. Part I. Chemosphere $\quad 75, \quad 347-354 . \quad$ doi:10.1016/j.position.2008.11.08610.1016/ j.chemosphere.2008.12.006

Lanzky, P. F., and Halling-Sørensen, B. (1998). The Toxic Effect of the Antibiotic Metronidazole on Aquatic Organisms. Chemosphere 35, 2553-2561. doi:10.1016/S0045-6535(97)00324-X

Le, T. X. H., Nguyen, T. V., Yacouba, Z. A., Zoungrana, L., Avril, F., Petit, E., et al. (2016). Toxicity Removal Assessments Related to Degradation Pathways of Azo Dyes: Toward an Optimization of Electro-Fenton Treatment. Chemosphere 161, 308-318. doi:10.1016/j.chemosphere.2016.06.108

Lee, S. S., and Carlson, K. (2006). Occurrence of Ionophore Antibiotics in Water and Sediments of a Mixed-Landscape Watershed. Water Res. 40, 2549-2560. doi:10.1016/j.watres.2006.04.036

Li, D., Zeng, S., He, M., and Gu, A. Z. (2016). Water Disinfection Byproducts Induce Antibiotic Resistance-Role of Environmental Pollutants in Resistance Phenomena. Environ. Sci. Technol. 50, 3193-3201. doi:10.1021/acs.est.5b05113

Li, F., Chen, L., Chen, W., Bao, Y., Zheng, Y., Huang, B., et al. (2020b). Antibiotics in Coastal Water and Sediments of the East China Sea: Distribution, Ecological Risk Assessment and Indicators Screening. Mar. Pollut. Bull. 151, 110810. doi:10.1016/j.marpolbul.2019.110810

Li, P., Wu, Y., He, Y., Zhang, B., Huang, Y., Yuan, Q., et al. (2020a). Occurrence and Fate of Antibiotic Residues and Antibiotic Resistance Genes in a Reservoir with Ecological Purification Facilities for Drinking Water Sources. Sci. Total Environ. 707, 135276. doi:10.1016/j.scitotenv.2019.135276

Li, S., Shi, W., Li, H., Xu, N., Zhang, R., Chen, X., et al. (2018). Antibiotics in Water and Sediments of Rivers and Coastal Area of Zhuhai City, Pearl River Estuary, south China. Sci. Total Environ. 636, 1009-1019. doi:10.1016/ j.scitotenv.2018.04.358

Li, W., Shi, Y., Gao, L., Liu, J., and Cai, Y. (2012b). Investigation of Antibiotics in Mollusks from Coastal Waters in the Bohai Sea of China. Environ. Pollut. 162, 56-62. doi:10.1016/j.envpol.2011.10.022

Li, W., Shi, Y., Gao, L., Liu, J., and Cai, Y. (2012a). Occurrence of Antibiotics in Water, Sediments, Aquatic Plants, and Animals from Baiyangdian Lake in North China. Chemosphere 89, 1307-1315. doi:10.1016/ j.chemosphere.2012.05.079

Lian, L. (2016). Distribution of Antibiotics in Surface and Core Sediments from Aquaculture. Dalian, China: Areas Dalian University of Technology.

Liang, D. H., and Hu, Y. (2021). Application of a Heavy Metal-Resistant Achromobacter Sp. For the Simultaneous Immobilization of Cadmium and Degradation of Sulfamethoxazole from Wastewater. J. Hazard. Mater. 402, 124032. doi:10.1016/j.jhazmat.2020.124032

Liang, D. H., Hu, Y., Cheng, J., and Chen, Y. (2020). Simultaneous Sulfamethoxazole Biodegradation and Nitrogen Conversion in Low $\mathrm{C} / \mathrm{N}$ Ratio Pharmaceutical Wastewater by Achromobacter Sp. JL9. Sci. Total Environ. 703, 135586. doi:10.1016/j.scitotenv.2019.135586

Liang, S. (2019). Distribution Characteristics and Risk Assessment of Antibiotics in Lancang and Yarlung Zangbo Rivers. Beijing, China: China University of Geosciences.

Liao, J., Wei, X. Q., Xiao, Y. Q., Li, Q. S., Fan, H. Y., Liu, X. J., et al. (2020). [Pollution Characteristics and Risk Assessment of Antibiotics in Lianhua Reservoir]. Huan Jing Ke Xue 41, 4081-4087. doi:10.13227/j.hjkx.202002084

Limbu, S. M., Zhou, L., Sun, S.-X., Zhang, M.-L., and Du, Z.-Y. (2018). Chronic Exposure to Low Environmental Concentrations and Legal Aquaculture Doses of Antibiotics Cause Systemic Adverse Effects in Nile tilapia and Provoke Differential Human Health Risk. Environ. Int. 115, 205-219. doi:10.1016/ j.envint.2018.03.034

Liu, J., Lu, G., Wang, Y., Yan, Z., Yang, X., Ding, J., et al. (2014). Bioconcentration, Metabolism, and Biomarker Responses in Freshwater Fish Carassius auratus Exposed to Roxithromycin. Chemosphere 99, 102-108. doi:10.1016/ j.chemosphere.2013.10.036

Liu, J., Lu, G., Yang, X., and Jin, S. (2012). Distribution, Accumulation and EcoToxicological Effects of Antibiotics in Aquatic Environment. Adm. Tech. Environ. Monit. 24, 14-20.

Liu, L., Liu, C., Zheng, J., Huang, X., Wang, Z., Liu, Y., et al. (2013). Elimination of Veterinary Antibiotics and Antibiotic Resistance Genes from Swine Wastewater in the Vertical Flow Constructed Wetlands. Chemosphere 91, 1088-1093. doi:10.1016/j.chemosphere.2013.01.007

Liu, M., Zhou, Z., Liu, Y., Zhao, J., and Cai, Y. (2017). Distribution Characteristics of Antibiotics in Part of Pearl River. Guangzhou Chem. Industry.

Liu, S., Zhang, L., Li, H., Chen, M., Chen, L., Wang, J., et al. (2020b). Occurrence, Distribution and Ecological Risk Assessment of Antibiotics in Sediments of Minjiang Estuary. J. Oper. Oceanogr. 39, 162-171. doi:10.3969/J.ISSN.20954972.2020.02.002

Liu, T., Lun, J., Zheng, P., Feng, J., Meng, S., Peng, T., et al. (2020c). Diversity and Distribution of Antibiotics and Antibiotic Resistance Genes in Seven National Mangrove Nature Reserves, South China. Int. Biodeterioration Biodegradation 153, 105000. doi:10.1016/j.ibiod.2020.105000

Liu, X., Guo, X., Liu, Y., Lu, S., Xi, B., Zhang, J., et al. (2019). A Review on Removing Antibiotics and Antibiotic Resistance Genes from Wastewater by Constructed Wetlands: Performance and Microbial Response. Environ. Pollut. 254, 112996. doi:10.1016/j.envpol.2019.112996

Liu, X., Lu, S., Guo, W., Xi, B., and Wang, W. (2018). Antibiotics in the Aquatic Environments: A Review of Lakes, China. Sci. Total Environ. 627, 1195-1208. doi:10.1016/j.scitotenv.2018.01.271

Liu, X., and Lu, S. (2018). Occurrence and Ecological Risk of Typical Antibiotics in Surface Water of the Datong Lake, China China Environ. Sci 38, 320-329. doi:10.19674/j.cnki.issn1000-6923.2018.0038

Liu, Y., Feng, M., Wang, B., Zhao, X., Guo, R., Bu, Y., et al. (2020a). Distribution and Potential Risk Assessment of Antibiotic Pollution in the Main Drinking Water Sources of Nanjing, China. Environ. Sci. Pollut. Res. 27, 21429-21441. doi:10.1007/s11356-020-08516-7

Liu, Y., Li, Z., Feng, Y., Cheng, D., Hu, H., and Zhang, W. (2016). Research Progress in Microbial Degradation of Antibiotics. J. Agro-environ. Sci. 35, 212-224.

Liyanage, G. Y., and Manage, P. M. (2018). Removal of Ciprofloxacin (CIP) by Bacteria Isolated from Hospital Effluent Water and Identification of Degradation Pathways. Ijmpd 2, 37-47. doi:10.22161/ijmpd.2.3.1

Llorca, M., Rodríguez-Mozaz, S., Couillerot, O., Panigoni, K., de Gunzburg, J., Bayer, S., et al. (2015). Identification of New Transformation Products during Enzymatic Treatment of Tetracycline and Erythromycin Antibiotics at Laboratory Scale by an On-Line Turbulent Flow Liquid-Chromatography Coupled to a High Resolution Mass Spectrometer LTQ-Orbitrap. Chemosphere 119, 90-98. doi:10.1016/j.chemosphere.2014.05.072

Loftin, K. A., Adams, C. D., Meyer, M. T., and Surampalli, R. (2008). Effects of Ionic Strength, Temperature, and $\mathrm{pH}$ on Degradation of Selected Antibiotics. J. Environ. Qual. 37, 378-386. doi:10.2134/jeq2007.0230

Lü, L., Zhang, M. L., Wang, C. M., Lin, H. R., and Yu, X. (2015). [Effect of Three Typical Disinfection Byproducts on Bacterial Antibiotic Resistance]. Huan Jing Ke Xue 36, 2525-2531. doi:10.13227/j.hjkx.2015.07.027

Lv, L., Yu, X., Xu, Q., and Ye, C. (2015). Induction of Bacterial Antibiotic Resistance by Mutagenic Halogenated Nitrogenous Disinfection Byproducts. Environ. Pollut. 205, 291-298. doi:10.1016/j.envpol.2015.06.026

Lyu, K., Liu, X., Deng, C., Zheng, K., Li, L., Shi, J., et al. (2019). Determination of 14 Antibiotics in Water and Sediment by SPE-RRLC-MS/MS. Environ. Chem. 38, 2415-2424. doi:10.7524/j.issn.0254-6108.2018122904

Madan, J. C., Salari, R. C., Saxena, D., Davidson, L., O’Toole, G. A., Moore, J. H., et al. (2012). Gut Microbial Colonisation in Premature Neonates Predicts Neonatal Sepsis. Arch. Dis. Child. Fetal Neonatal. Ed. Heart 201297 (6), F456-F462. doi:10.1136/fetalneonatal-2011-301373

Maia, A. S., Tiritan, M. E., and Castro, P. M. L. (2018). Enantioselective Degradation of Ofloxacin and Levofloxacin by the Bacterial Strains Labrys Portucalensis F11 and Rhodococcus Sp. FP1. Ecotoxicology Environ. Saf. 155, 144-151. doi:10.1016/j.ecoenv.2018.02.067

Mangalgiri, K. P., and Blaney, L. (2017). Elucidating the Stimulatory and Inhibitory Effects of Dissolved Organic Matter from Poultry Litter on Photodegradation of Antibiotics. Environ. Sci. Technol. 51, 12310-12320. doi:10.1021/ acs.est.7b03482

Mao, F., Liu, X., Wu, K., Zhou, C., and Si, Y. (2018). Biodegradation of Sulfonamides by Shewanella Oneidensis MR-1 and Shewanella Sp. Strain MR-4. Biodegradation 29, 129-140. doi:10.1007/s10532-0179818-5

Marcelo, C., Susana, R., and Augusto, S. (2020). [Use (and abuse) of antibiotics in perinatal medicine]Anales de pediatria. Barcelona, Spain. 
Martins, M., Sanches, S., and Pereira, I. A. C. (2018). Anaerobic Biodegradation of Pharmaceutical Compounds: New Insights into the Pharmaceutical-Degrading Bacteria. J. Hazard. Mater. 357, 289-297. doi:10.1016/j.jhazmat.2018.06.001

Matamoros, V., and Bayona, J. M. (2006). Elimination of Pharmaceuticals and Personal Care Products in Subsurface Flow Constructed Wetlands. Environ. Sci. Technol. 40, 5811-5816. doi:10.1021/es0607741

Mcinnes, R. S., Mccallum, G. E., Lamberte, L. E., and Van Schaik, W. (2020). Horizontal Transfer of Antibiotic Resistance Genes in the Human Gut Microbiome. Curr. Opin. Microbiol. 53, 35-43. doi:10.1016/j.mib.2020.02.002

Meng, Y., Feng, Y., Li, X., Liu, Y., and Li, Z. (2018). Isolation of an OxytetracyclineDegrading Bacterial Strain and its Biodegradation Characteristics. Plant Nutr. Fert. Sci. 24, 720-727. doi:10.11674/zwyf.17161

Metsälä, J., Lundqvist, A., Virta, L. J., Kaila, M., Gissler, M., and Virtanen, S. M. (2015). Prenatal and post-natal Exposure to Antibiotics and Risk of Asthma in Childhood. Clin. Exp. Allergy 45, 137-145. doi:10.1111/cea.12356

Mette, N., Vera, E., Beck, N. R., Sigmund, B. L., Toft, S. H., and Ratner, A. J. (2012). Maternal Use of Antibiotics, Hospitalisation for Infection during Pregnancy, and Risk of Childhood Epilepsy: A Population-Based Cohort Study. Plos One 7, e30850.

Milliken, S., Allen, R. M., and Lamont, R. F. (2019). The Role of Antimicrobial Treatment during Pregnancy on the Neonatal Gut Microbiome and the Development of Atopy, Asthma, Allergy and Obesity in Childhood. Expert Opin. Drug Saf. 18, 173-185. doi:10.1080/14740338.2019.1579795

Möhle, L., Mattei, D., Heimesaat, M. M., Bereswill, S., Fischer, A., Alutis, M., et al. (2016). Ly6Chi Monocytes Provide a Link between Antibiotic-Induced Changes in Gut Microbiota and Adult Hippocampal Neurogenesis. Cel Rep. 15, 1945-1956. doi:10.1016/j.celrep.2016.04.074

Mosedale, M., Wu, H., Kurtz, C. L., Schmidt, S. P., Adkins, K., and Harrill, A. H. (2014). Dysregulation of Protein Degradation Pathways May Mediate the Liver Injury and Phospholipidosis Associated with a Cationic Amphiphilic Antibiotic Drug. Toxicol. Appl. Pharmacol. 280, 21-29. doi:10.1016/j.taap.2014.06.013

Mountassir, E. M., Benaziz, L., Rafqah, S., and Lakraimi, M. (2020). Development of a New Recyclable Nanocomoposite LDH-TiO2 for the Degradation of Antibiotic Sulfamethoxazole under UVA Radiation: an Approach towards Sunlight. J. Photoch. Photobio. A. 396, 112530. doi:10.1016/ j.jphotochem. 2020.112530

Mulla, S. I., Hu, A., Sun, Q., Li, J., Suanon, F., Ashfaq, M., et al. (2018). Biodegradation of Sulfamethoxazole in Bacteria from Three Different Origins. J. Environ. Manage. 206, 93-102. doi:10.1016/j.jenvman.2017.10.029

Mushtaq, K., Saeed, M., Gul, W., Munir, M., Firdous, A., Yousaf, T., et al. (2020). Synthesis and Characterization of $\mathrm{TiO} 2$ via Sol-Gel Method for Efficient Photocatalytic Degradation of Antibiotic Ofloxacin. Inorg. Nano-Metal Chem. 50, 580-586. doi:10.1080/24701556.2020.1722695

Nassar, R., Rifai, A., Trivella, A., Mazellier, P., Mokh, S., and Al-Iskandarani, M. (2018). Aqueous Chlorination of Sulfamethazine and Sulfamethoxypyridazine: Kinetics and Transformation Products Identification. J. Mass. Spectrom. 53, 614-623. doi:10.1002/jms.4191

Neu, J., and Walker, W. A. (2011). Necrotizing Enterocolitis. N. Engl. J. Med. 364, 255-264. doi:10.1056/nejmra1005408

Nguyen, L. N., Nghiem, L. D., and Oh, S. (2018). Aerobic Biotransformation of the Antibiotic Ciprofloxacin by Bradyrhizobium Sp. Isolated from Activated Sludge. Chemosphere 211, 600-607. doi:10.1016/ j.chemosphere.2018.08.004

Pan, L.-J., Li, J., Li, C.-x., Tang, X.-d., Yu, G.-W., and Wang, Y. (2018). Study of Ciprofloxacin Biodegradation by a Thermus Sp. Isolated from Pharmaceutical Sludge. J. Hazard. Mater. 343, 59-67. doi:10.1016/j.jhazmat.2017.09.009

Park, N., Vanderford, B. J., Snyder, S. A., Sarp, S., Kim, S. D., and Cho, J. (2009). Effective Controls of Micropollutants Included in Wastewater Effluent Using Constructed Wetlands under Anoxic Conditionuent Using Constructed Wetlands under Anoxic Condition. Ecol. Eng. 35, 418-423. doi:10.1016/ j.ecoleng.2008.10.004

Park, Y. J., Chang, J., Lee, G., Son, J. S., and Park, S. M. (2020). Association of Class Number, Cumulative Exposure, and Earlier Initiation of Antibiotics during the First Two-Years of Life with Subsequent Childhood Obesity. Metabolism 112, 154348. doi:10.1016/j.metabol.2020.154348

Peng, Y., Zhang, Y., Huang, H., and Zhong, C. (2018). Flexibility Induced HighPerformance MOF-Based Adsorbent for Nitroimidazole Antibiotics Capture. Chem. Eng. J. 333, 678-685. doi:10.1016/j.cej.2017.09.138
Pérez, S., and Barceló, D. (2008). First Evidence for Occurrence of Hydroxylated Human Metabolites of Diclofenac and Aceclofenac in Wastewater Using QqLIT-MS and QqTOF-MS. Anal. Chem. 80, 8135-8145. doi:10.1021/ ac801167w

Pérez-Grisales, M. S., Castrillón-Tobón, M., Copete-Pertuz, L. S., Plácido, J., and Mora-Martínez, A. L. (2019). Biotransformation of the Antibiotic Agent Cephadroxyl and the Synthetic Dye Reactive Black 5 by Leptosphaerulina Sp. Immobilised on Luffa (Luffa Cylindrica) Sponge. Biocatal. Agric. Biotechnol. 18, 101051. doi:10.1016/j.bcab.2019.101051

Pronovost, G. N., and Hsiao, E. Y. (2019). Perinatal Interactions between the Microbiome, Immunity, and Neurodevelopment. Immunity 50, 18-36. doi:10.1016/j.immuni.2018.11.016

Qian, Z., Tang, S., Liang, Y., Wei, S., Luo, F., and Chen, S. (2019). Simultaneous Determination of Sulfonamides, Quinolones and Macrolides Anbiotics Residues in Sediment from Aquaculture Environment by QuECHERs-HPLC-MS/MS. J. Chin. Mass. Spectrom. Soc. 40, 356-368. doi:10.7538/zpxb.2018.0128

Qianqian, W. (2016). Pollution Levels of Antibiotics from Aquatic Environment in Alashankou Region of Xinjiang and Surrounding Area. Shihezi, China: Shihezi University. doi:10.1109/ccdc.2016.7531705

Qing, C., Shang, C., Zhou, Y., Lin, Y., Shao, J., and Chen, A. (2018). Study on the Biodegradation of Tetracycline Wastewater by Phanerochaete Chrysoporium. Environ. Pollut. Control. 40, 1023-1026+1067. doi:10.15985/j.cnki.10013865.2018.09.014

Radjenovic, J., Petrovic, M., and Barcelo, D. (2009). Fate and Distribution of Pharmaceuticals in Wastewater and Sewage Sludge of the Conventional Activated Sludge (CAS) and Advanced Membrane Bioreactor (MBR) Treatment. Water Res. 43, 831-841. doi:10.1016/j.watres.2008.11.043

Radke, M., Lauwigi, C., Heinkele, G., Mürdter, T. E., and Letzel, M. (2009). Fate of the Antibiotic Sulfamethoxazole and its Two Major Human Metabolites in a Water Sediment Test. Environ. Sci. Technol. 43, 3135-3141. doi:10.1021/ es 900300 u

Ramirez, A. J., Mottaleb, M. A., Brooks, B. W., and Chambliss, C. K. (2007). Analysis of Pharmaceuticals in Fish Using Liquid Chromatography-Tandem Mass Spectrometry. Anal. Chem. 79, 3155-3163. doi:10.1021/ac062215i

Reis, P. J. M., Homem, V., Alves, A., Vilar, V. J. P., Manaia, C. M., and Nunes, O. C. (2018). Insights on Sulfamethoxazole Bio-Transformation by Environmental Proteobacteria Isolates. J. Hazard. Mater. 358, 310-318. doi:10.1016/ j.jhazmat.2018.07.012

Rico, A., Phu, T. M., Satapornvanit, K., Min, J., Shahabuddin, A. M., Henriksson, P. J. G., et al. (2013). Use of Veterinary Medicines, Feed Additives and Probiotics in Four Major Internationally Traded Aquaculture Species Farmed in Asia. Aquaculture 412-413, 231-243. doi:10.1016/j.aquaculture.2013.07.028

Russell, J. N., and Yost, C. K. (2021). Alternative, Environmentally Conscious Approaches for Removing Antibiotics from Wastewater Treatment Systems. Chemosphere 263, 128177. doi:10.1016/j.chemosphere.2020.128177128177

Scott, F. I., Horton, D. B., Mamtani, R., Haynes, K., Goldberg, D. S., Lee, D. Y., et al. (2016). Administration of Antibiotics to Children before Age 2 Years Increases Risk for Childhood Obesity. Gastroenterology 151, 120-129. doi:10.1053/ j.gastro.2016.03.006

Shakerian, F., Zhao, J., and Li, S.-P. (2020). Recent Development in the Application of Immobilized Oxidative Enzymes for Bioremediation of Hazardous Micropollutants - A Review. Chemosphere 239, 124716. doi:10.1016/ j.chemosphere.2019.124716

Shao, B., Liu, Z., Zeng, G., Liu, Y., Yang, X., Zhou, C., et al. (2019). Immobilization of Laccase on Hollow Mesoporous Carbon Nanospheres: Noteworthy Immobilization, Excellent Stability and Efficacious for Antibiotic Contaminants Removal. J. Hazard. Mater. 362, 318-326. doi:10.1016/ j.jhazmat.2018.08.069

Shao, S., Hu, Y., Cheng, C., Cheng, J., and Chen, Y. (2018a). Simultaneous Degradation of Tetracycline and Denitrification by a Novel Bacterium, Klebsiella Sp. SQY5. Chemosphere 209, 35-43. doi:10.1016/ j.chemosphere.2018.06.093

Shao, S., Hu, Y., Cheng, J., and Chen, Y. (2018b). Degradation of Oxytetracycline (OTC) and Nitrogen Conversion Characteristics Using a Novel Strain. Chem. Eng. J. 354, 758-766. doi:10.1016/j.cej.2018.08.032

Shao, Z., Li, H., Li, X., Xu, Y., and Zheng, X. (2020). The Occurrence and Risk Management of Antibiotics and Antibiotic Resistant Genes in Rural Solid Waste. Asian J. Org. Chem. 15, 112-122. 
Shi, M., Zhu, X., and Tang, W. (2015). Progress in Research of the Environmental Effects of Antibiotic Resistance Genes. Environ. Prot. Sci. 41, 123-128. doi:10.16803/j.cnki.issn.1004-6216.2015.06.027

Simón-Herrero, C., Naghdi, M., Taheran, M., Kaur Brar, S., Romero, A., Valverde, J. L., et al. (2019). Immobilized Laccase on Polyimide Aerogels for Removal of Carbamazepine. J. Hazard. Mater. 376, 83-90. doi:10.1016/j.jhazmat.2019.05.032

Singh, S. K., Khajuria, R., and Kaur, L. (2017). Biodegradation of Ciprofloxacin by white Rot Fungus Pleurotus Ostreatus. 3 Biotech. 7, 7. doi:10.1007/s13205-0170684-y

Siswanto, S., Arozal, W., Juniantito, V., Grace, A., Agustini, F. D., and Nafrialdi, N. (2016). The Effect of Mangiferin against Brain Damage Caused by Oxidative Stress and Inflammation Induced by Doxorubicin. HAYATI J. Biosciences 23, 51-55. doi:10.1016/j.hjb.2016.02.001

Sobhani, I., Bergsten, E., Couffin, S., Amiot, A., Nebbad, B., Barau, C., et al. (2019). Colorectal Cancer-Associated Microbiota Contributes to Oncogenic Epigenetic Signatures. Proc. Natl. Acad. Sci. USA 116, 24285-24295. doi:10.1073/ pnas. 1912129116

Sun, Q., Wang, Z., Dong, J., Chen, C., Chen, Q., Liu, J., et al. (2018). Spatialtemporal Distribution and Risk Evaluation of Four Typical Antibiotics in River Networks of Taihu Lake Basin. Acta Scientiae Circumstantiae. 38, 4400-4410. doi:10.13671/j.hjkxxb.2018.0212

Susarla, S., Medina, V. F., and McCutcheon, S. C. (2002). Phytoremediation: An Ecological Solution to Organic Chemical Contamination. Ecol. Eng. 18, 647-658. doi:10.1016/S0925-8574(02)00026-5

Tadeusz, P., Rafał, K., Alicja, B., and Zbigniew, S. (2019). [The Growing Resistance of Bacterial Strains to Antibiotics]. Polski Merkuriusz Lekarski : Organ. Polskiego Towarzystwa Lekarskiego 47 (279), 106-110.

Tang, J., Shi, T., Wu, X., Cao, H., Li, X., Hua, R., et al. (2015). The Occurrence and Distribution of Antibiotics in Lake Chaohu, China: Seasonal Variation, Potential Source and Risk Assessment. Chemosphere 122, 154-161. doi:10.1016/j.chemosphere.2014.11.032

Tang, J., Wang, S., Tai, Y., Tam, N. F., Su, L., Shi, Y., et al. (2020). Evaluation of Factors Influencing Annual Occurrence, Bioaccumulation, and Biomagnification of Antibiotics in Planktonic Food Webs of a Large Subtropical River in South China. Water Res. 170, 115302. doi:10.1016/ j.watres.2019.115302

Terzic, S., Udikovic-Kolic, N., Jurina, T., Krizman-Matasic, I., Senta, I., Mihaljevic, I., et al. (2018). Biotransformation of Macrolide Antibiotics Using Enriched Activated Sludge Culture: Kinetics, Transformation Routes and Ecotoxicological Evaluation. J. Hazard. Mater. 349, 143-152. doi:10.1016/ j.jhazmat.2018.01.055

Touahar, I. E., Haroune, L., Ba, S., Bellenger, J.-P., and Cabana, H. (2014). Characterization of Combined Cross-Linked Enzyme Aggregates from Laccase, Versatile Peroxidase and Glucose Oxidase, and Their Utilization for the Elimination of Pharmaceuticals. Sci. Total Environ. 481, 90-99. doi:10.1016/j.scitotenv.2014.01.132

Tunç, S., Duman, O., and Gürkan, T. (2013). Monitoring the Decolorization of Acid Orange 8 and Acid Red 44 from Aqueous Solution Using Fenton's Reagents by Online Spectrophotometric Method: Effect of Operation Parameters and Kinetic Study. Ind. Eng. Chem. Res. 52, 1414-1425. doi:10.1021/ie302126c

Tunç, S., Gürkan, T., and Duman, O. (2012). On-Line Spectrophotometric Method for the Determination of Optimum Operation Parameters on the Decolorization of Acid Red 66 and Direct Blue 71 from Aqueous Solution by Fenton Process. Chem. Eng. J. 181-182, 431-442. doi:10.1016/j.cej.2011.11.109

Wang, D., and Wang, Q. (2020). Analysis on the Distribution of Antibiotics Pollution in the Water Environment of Weihe River Area in Huaihe River Basin. Environ. Sci. Technol. 45, 63-66.

Wang, G., Zhou, S., Han, X., Zhang, L., Ding, S., li, Y., et al. (2020b). Occurrence, Distribution, and Source Track of Antibiotics and Antibiotic Resistance Genes in the Main Rivers of Chongqing City, Southwest China. J. Hazard. Mater. 389, 122110. doi:10.1016/j.jhazmat.2020.122110

Wang, J. (2020a). Removal of Pharmaceuticals and Personal Care Products (PPCPs) from Eastewater: a Review. J. Sichuan Norm. Univ. Nat. Sci. 43, $143-172$.

Wang, M., and Helbling, D. E. (2016). A Non-target Approach to Identify Disinfection Byproducts of Structurally Similar Sulfonamide Antibiotics. Water Res. 102, 241-251. doi:10.1016/j.watres.2016.06.042
Wang, Q., Zhu, P., Xia, Z., Wang, Z., Zeng, Y., and Hou, Y. (2018b). Screening and Degradation Properties of Three Kinds of Agricultural Antibiotics Degrading Fungi. J. Agric. Resour. Environ. 35, 533-539. doi:10.13254/j.jare.2018.0069

Wang, R., QiuQian, L., Li, G., Zong, Y., Tang, J., and Xu, Y. (2018d). Distribution Characteristics and Ecological Risk Assessment of Selected Antibiotics in Moon Lake, Ningbo City. J. Lake Sci. 30, 1616-1624. doi:10.18307/2018.0613

Wang, S., Hu, Y., and Wang, J. (2018a). Biodegradation of Typical Pharmaceutical Compounds by a Novel Strain Acinetobacter Sp. J. Environ. Manage. 217, 240-246. doi:10.1016/j.jenvman.2018.03.096

Wang, S., and Wang, J. (2018). Biodegradation and Metabolic Pathway of Sulfamethoxazole by a Novel Strain Acinetobacter Sp. Appl. Microbiol. Biotechnol. 102, 425-432. doi:10.1007/s00253-017-8562-4

Wang, T., Hu, X., Liang, S., Li, W., Wu, X., Wang, L., et al. (2015). Lactobacillus Fermentum NS9 Restores the Antibiotic Induced Physiological and Psychological Abnormalities in Rats. Beneficial Microbes 6, 707-717. doi:10.3920/bm2014.0177

Wang, W., Zhang, W., Liang, H., and Gao, D. (2018c). Seasonal Distribution Characteristics and Health Risk Assessment of Typical Antibiotics in the Harbin Section of the Songhua River basin. Environ. Technology 40, 2726-2737. doi:10.1080/09593330.2018.1449902

Wang, Y. (2020b). Distribution Characteristics of Typical Antibiotics, Antibiotic Resistance Genes and Microbial Community in Ebinur Lake Basin. Ji'nan, China: Shandong Normal University.

Wang, Y., Huang, H., Peng, J., Xie, S., Yang, H., Guo, F., et al. (2020a). Occurrence and Distribution of Typical Antibiotics in the Aquatic Environment of the Wetland Karst Plateau in Guizhou. Environ. Chem. 39, 975-986. doi:10.7524/ j.issn.0254-6108.2019090103

Wang, Y., Jia, H., Zhang, H., Wang, J., and Liu, W. (2017a). Performance of a Novel Recycling Magnetic Flocculation Membrane Filtration Process for Tetracycline-Polluted Surface Water Treatment. Water Sci. Technol. 76, 490-500. doi:10.2166/wst.2017.218

Wang, Y., Peng, J., Huang, H., Tan, H., Zhang, A., Yang, H., et al. (2018e). Distribution Characteristics of Typical Antibiotics in Urban Rivers of Guiyang City. Environ. Chem. 37, 2039-2048.

Wang, Z., Du, Y., Yang, C., Liu, X., Zhang, J., Li, E., et al. (2017b). Occurrence and Ecological hazard Assessment of Selected Antibiotics in the Surface Waters in and Around Lake Honghu, China. Sci. Total Environ. 609, 1423-1432. doi:10.1016/j.scitotenv.2017.08.009

Wang, Z., Lei, Y., Xiao, j., Luo, Z., Zhong, H., Guo, Z., et al. (2019). Residue Status of Antibiotics in Aquaculture Ponds of Main tilapia Aquaculture Areas in Guangxi. J. South. Agric. 50, 891-897. doi:10.3969/j.issn.2095-1191.2019.04.29

Wei, R., He, T., Zhang, S., Zhu, L., Shang, B., Li, Z., et al. (2019). Occurrence of Seventeen Veterinary Antibiotics and Resistant Bacterias in Manure-Fertilized Vegetable Farm Soil in Four Provinces of China. Chemosphere 215, 234-240. doi:10.1016/j.chemosphere.2018.09.152

Wen, X., Jia, Y., and Li, J. (2009). Degradation of Tetracycline and Oxytetracycline by Crude Lignin Peroxidase Prepared from Phanerochaete Chrysosporium - A white Rot Fungus. Chemosphere 75, 1003-1007. doi:10.1016/ j.chemosphere.2009.01.052

Weng, S.-S., Ku, K.-L., and Lai, H.-T. (2012). The Implication of Mediators for Enhancement of Laccase Oxidation of Sulfonamide Antibiotics. Bioresour. Technology 113, 259-264. doi:10.1016/j.biortech.2011.12.111

Weng, X., Owens, G., and Chen, Z. (2020). Synergetic Adsorption and Fenton-like Oxidation for Simultaneous Removal of Ofloxacin and Enrofloxacin Using green Synthesized Fe NPs. Chem. Eng. J. 382, 122871. doi:10.1016/ j.cej.2019.122871

Winek, K., Engel, O., Koduah, P., Heimesaat, M. M., Fischer, A., Bereswill, S., et al. (2016). Depletion of Cultivatable Gut Microbiota by Broad-Spectrum Antibiotic Pretreatment Worsens Outcome after Murine Stroke. Stroke 47, 1354-1363. doi:10.1161/strokeaha. 115.011800

Wu, X., Wu, X., Li, J., Shen, L., Yu, R., and Zeng, W. (2018). Isolation and Degradation Characteristics of a Efficient Tetracyclinedegrading Strain. Biotechnol. Bull. 34, 172-178. doi:10.13560/j.cnki.biotech.bull.1985.2017-0723

Wu, X. Y., Zou, H., Zhu, R., and Wang, J. G. (2016). [Occurrence, Distribution and Ecological Risk of Aantibiotics in Surface Water of the Gonghu Bay, Taihu Lake]. Huan Jing Ke Xue 37, 4596-4604. doi:10.13227/j.hjkx.201603005

Xian, Q., Hu, L., Chen, H., Chang, Z., and Zou, H. (2010). Removal of Nutrients and Veterinary Antibiotics from Swine Wastewater by a Constructed 
Macrophyte Floating Bed System. J. Environ. Manage. 91, 2657-2661. doi:10.1016/j.jenvman.2010.07.036

Xiao, X., Wu, Y., Ding, H., Wan, L., Yang, W., and Zhang, W. (2019). Pollution Characteristics of Antibiotics and Antibiotic Resistance Genes in Urban Lakes of Wuhan. Environ. Sci. Technol. 42, 9-16. doi:10.19672/j.cnki.10036504.2019.03.002

Xie, C., Yang, S.-t., Wei, Q., Jiang, X., Wang, Z., and Wu, X. (2019). Antibiotic Pollution Characteristics and Risk Assessment of Xinghu Lake in Zhaoqing. J. Environ. Health 36, 427-431. doi:10.16241/j.cnki.1001-5914.2019.05.012

Xie, Q., Chen, Y., Wan, J., Wang, Y., and Yan, Z. (2020). Occurrence, Distribution and Risk Assessment of Antibiotics in Drinking Water Source in Dongguan. ActaScientiae Circumstantiae 40, 166-178. doi:10.13671/j.hjkxxb.2019.0334

Xingxing, L., Jianguo, B., Yifei, L., Ying, L., Hui, G., and Jin, Z. (2016). Degradation Mechanism of Tetracycline by Lignin Peroxidase. Saf. Environ. Eng. 23, 61-68. doi:10.13578/j.cnki.issn.1671-1556.2016.05.01010.31723/2524-0447-2016-23193-204

Xu, B., Luo, Y., Zhou, Q., and Mao, D. (2010). Sources, Dissemination, and Ecological Risk of Antibiotic Resistances Genes(ARGs) in the Environment. Environ. Chem. 29, 169-178.

Xu, L., Sun, B., Sheng, P., Shi, Q., and Luo, Y. (2019). Pollution Characteristics of Antibacterial Drugs in Typical Aquaculture Ponds in Huzhou Area. Jiangsu Agric. Sci. 47, 210-214. doi:10.15889/j.issn.1002-1302.2019.11.047

Xu, L., Ye, X., Hao, G., Sheng, P., Zhou, D., Sun, B., et al. (2020). Ypical Antibiotic Pollution Characteristics and Ecological Risk Assessment of Surface Water in Tiaoxi River. Mod. Agr. Sci. Tech. 180, 183+187.

Xu, Z., Li, T., Bi, J., and Wang, C. (2018). Spatiotemporal Heterogeneity of Antibiotic Pollution and Ecological Risk Assessment in Taihu Lake Basin, China. Sci. Total Environ. 643, 12-20. doi:10.1016/j.scitotenv.2018.06.175

Xuan, L., Huimin, Z., Hua, W., Lu, L., Shihui, W., and Qi, S. (2020). Impacts of Heavy Metals and Environmental Factors Assisted Antibiotics on Antibiotic Resistance Genes in Sediments in Dalian Typical Intertidal Mudflat Culture Areas. J. Dalian Ocean Univ. 35, 229-238. doi:10.16535/ j.cnki.dlhyxb.2020-012

Yamaguchi, K., Beligni, M. V., Prieto, S., Haynes, P. A., McDonald, W. H., Yates, J. R., et al. (2003). Proteomic Characterization of the Chlamydomonas Reinhardtii Chloroplast Ribosome. J. Biol. Chem. 278, 33774-33785. doi:10.1074/jbc.M301934200

Yamaguchi, K., and Subramanian, A. R. (2003). Proteomic Identification of All Plastid-specific Ribosomal Proteins in Higher Plant Chloroplast 30S Ribosomal Subunit. PSRP-2 (U1A-type Domains), PSRP-3alpha/beta (Ycf65 Homologue) and PSRP-4 (Thx Homologue). Eur. J. Biochem. 270, 190-205. doi:10.1046/ j.1432-1033.2003.03359.x

Yan, R. (2019). Occurrence Characteristics and Risk Assessment of Antibiotics and Other Drugs in Shanghai Livestock Farms. Shanghai, China: East China Normal University.

Yan, X. (2018). Distribution, Sources and Risk Evaluation of Typical Antibiotics in Xiaoqing River Basin. Ji'nan, China: Shandong Normal University.

Yang, J.-F., Ying, G.-G., Zhao, J.-L., Tao, R., Su, H.-C., and Chen, F. (2010). Simultaneous Determination of Four Classes of Antibiotics in Sediments of the Pearl Rivers Using RRLC-MS/MS. Sci. Total Environ. 408, 3424-3432. doi:10.1016/j.scitotenv.2010.03.049

Yang, X.-L., Xu, J.-Y., Song, H.-L., Wang, X., and Li, T. (2020). Enhanced Removal of Antibiotics in Wastewater by Membrane Bioreactor with Addition of rice Straw. Int. Biodeterioration Biodegradation 148, 104868. doi:10.1016/ j.ibiod.2019.104868

Yang, Y. (2018). Concentration Levels and Distribution Characteristics of Various Antibiotics in Kaidu River and Kongque River in Bazhou Area. Shihezi, China: Xinjiang Shihezi University.

Yin, F., Ji, C., Dong, H., Tao, X., and Chen, Y. (2016). Research Progress on Effect of Antibiotic on Anaerobic Digestion Treatment in Animal Manure. J. Agr. Sci. Tech-iran 18, 171-177. doi:10.13304/j.nykjdb.2015.702

Yin, F., Lin, S., Zhou, X., Dong, H., and Zhan, Y. (2021). Fate of Antibiotics during Membrane Separation Followed by Physical-Chemical Treatment Processes. Sci. Total Environ. 759, 143520. doi:10.1016/ j.scitotenv.2020.143520

Yoshizaki, S., and Tomida, T. (2000). Principle and Process of Heavy Metal Removal from Sewage Sludge. Environ. Sci. Technol. 34, 1572-1575. doi:10.1021/es990979s
Yu, N., Fang, H., Hu, J., Wang, Z., Ding, C., Yuan, H., et al. (2020). Contamination Characteristics and Ecological Risk Assessment of Antibiotics in Four Typical Procambarus clarkii Aquaculture Environments in Jiangsu Province, China. J. Environ. Sci. (China) 39, 386-393. doi:10.11654/jaes.2019-0983

Yuan, J., Ni, M., Liu, M., Zheng, Y., and Gu, Z. (2019). Occurrence of Antibiotics and Antibiotic Resistance Genes in a Typical Estuary Aquaculture Region of Hangzhou Bay, China. Mar. Pollut. Bull. 138, 376-384. doi:10.1016/ j.marpolbul.2018.11.037

Zdarta, J., Jankowska, K., Bachosz, K., Kijeńska-Gawrońska, E., ZgołaGrześkowiak, A., Kaczorek, E., et al. (2020). A Promising Laccase Immobilization Using Electrospun Materials for Biocatalytic Degradation of Tetracycline: Effect of Process Conditions and Catalytic Pathways. Catal. Today 348, 127-136. doi:10.1016/j.cattod.2019.08.042

Zeng, J., Pan, Y., Yang, J., Hou, M., Zeng, Z., and Xiong, W. (2019). Metagenomic Insights into the Distribution of Antibiotic Resistome between the GutAssociated Environments and the Pristine Environments. Environ. Int. 126, 346-354. doi:10.1016/j.envint.2019.02.052

Zhang, C., Feng, Y., Liu, Y., Cheng, D., Zheng, Y., and Li, Z. (2018a). The Degradation of Typical Antibiotics and Their Effects on Soil Bacterial Diversity in Spinach Soil. Chin. Agric. Sci. 51, 3736-3749. doi:10.3864/ j.issn.0578-1752.2018.19.011

Zhang, C., You, S., Zhang, J., Qi, W., Su, R., and He, Z. (2020a). An Effective Iin-Ssitu Method for Laccase Immobilization: Excellent Activity, Effective Antibiotic Removal Rate and Low Potential Ecological Risk for Degradation Products. Bioresour. Technology 308, 123271. doi:10.1016/j.biortech.2020.123271

Zhang, H., Du, M., Jiang, H., Zhang, D., Lin, L., Ye, H., et al. (2014). Occurrence, Seasonal Variation and Removal Efficiency of Antibiotics and Their Metabolites in Wastewater Treatment Plants, Jiulongjiang River Basin, South China. Environ. Sci. Process. Impacts 17, 225-234. doi:10.1039/C4EM00457D

Zhang, H., Song, S., Jia, Y., Wu, D., and Lu, H. (2019b). Stress-responses of Activated Sludge and Anaerobic Sulfate-Reducing Bacteria Sludge under LongTerm Ciprofloxacin Exposure. Water Res. 164, 114964. doi:10.1016/ j.watres.2019.114964

Zhang, J., Peng, X., and Jia, X. (2019c). Isolation and Characterization of Highly Efficient Sulfamethazine-Degrading Bacterium Strain J2. Acta Scientiae Circumstantiae 39, 2919-2927. doi:10.13671/j.hjkxxb.2019.0096

Zhang, N., Li, M., and Liu, X. (2018b). Distribution and Transformation of Antibiotic Resistance Genes in Soil. China Environ. Sci. 38, 2609-2617. doi:10.19674/j.cnki.issn1000-6923.20180521.001

Zhang, R., Pei, J., Zhang, R., Wang, S., Zeng, W., Huang, D., et al. (2018c). Occurrence and Distribution of Antibiotics in Mariculture Farms, Estuaries and the Coast of the Beibu Gulf, China: Bioconcentration and Diet Safety of Seafood. Ecotoxicology Environ. Saf. 154, 27-35. doi:10.1016/j.ecoenv.2018.02.006

Zhang, Y., Chen, H., Jing, L., and Teng, Y. (2020b). Ecotoxicological Risk Assessment and Source Apportionment of Antibiotics in the Waters and Sediments of a Peri-Urban River. Sci. Total Environ. 731, 139128. doi:10.1016/j.scitotenv.2020.139128

Zhang, Y., Rong, C., Song, Y., Wang, Y., Pei, J., Tang, X., et al. (2017). Oxidation of the Antibacterial Agent Norfloxacin during Sodium Hypochlorite Disinfection of marine Culture Water. Chemosphere 182. doi:10.1016/j.chemosphere.2017.05.023

Zhang, Y., Wang, M., Zhang, D., Li, B., Bai, H., Zhang, H., et al. (2019a). Screening and Functional Identification of Cephalosporin Degrading Bacteria Achromobacter Sp. YF-1. J. Agr. Sci. Tech-iran 21, 112-119. doi:10.13304/ j.nykjdb.2019.0257

Zhang, Y., Yan, X., Sun, Y., Wu, H., and Lu, J. (2019). Current Situation of Antibiotic Abuse in China and its Residues Distribution in the Environment. Contemp. Chem. Ind. 48, 2660-2662.

Zhang, Y., Zhang, G., Wang, Y., Liu, X., Bi, B., Liu, X., et al. (2021). Occurrence and Ecological Risk of Typical Antibiotics in Surface Water of the Lake SayramXinjiang. J. Lake Sci. 33, 483-493.

Zhao, H., Liu, S., Chen, J., Jiang, J., Xie, Q., and Quan, X. (2015). Biological Uptake and Depuration of Sulfadiazine and Sulfamethoxazole in Common Carp (Cyprinus carpio). Chemosphere 120, 592-597. doi:10.1016/ j.Chemosphere.2014.09.075

Zhao, L., Tan, S., Zhang, P., and Huang, T. (2016b). Effects of Different Organic Carbon Sources on Removal Efficiency of Tetracyclines from Wastewater in Constructed Wetlands. Water Resour. Prot. 32, 70-74. doi:10.3880/j.issn.10046933.2016.06.011 
Zhao, R., Li, X., Hu, M., Li, S., Zhai, Q., and Jiang, Y. (2017). Efficient Enzymatic Degradation Used as Pre-stage Treatment for Norfloxacin Removal by Activated Sludge. Bioproc. Biosyst. Eng. 40, 1261-1270. doi:10.1007/s00449-017-1786-y

Zhao, S., Wang, X., Li, Y., and Lin, J. (2016a). Bioconcentration, Metabolism, and Biomarker Responses in marine Medaka (Oryzias Melastigma) Exposed to Sulfamethazine. Aquat. Toxicol. 181, 29-36. doi:10.1016/j.aquatox.2016.10.026

Zheng, C., Wu, H., Li, F., and Du, W. (2017). Water Quality Evaluation of Typical Freshwater Aquaculture Area in Zhejiang Province. J. Zhejiang Agric. Sci. 58, 2268-2274. doi:10.16178/j.issn.0528-9017.20171258

Zheng, H. (2017). Study on Distribution Characteristics and Health Risks of Some Antibiotics in Water Supply of a Water Plant, Huizhou City. Prev. Med. Tribune 23, 887-891. doi:10.16406/j.pmt.issn.1672-9153.2017.12.003

Zhong, Y., Chen, Z.-F., Dai, X., Liu, S.-S., Zheng, G., Zhu, X., et al. (2018). Investigation of the Interaction between the Fate of Antibiotics in Aquafarms and Their Level in the Environment. J. Environ. Manage. 207, 219-229. doi:10.1016/j.jenvman.2017.11.030

Zhou, W., Tang, Y., Du, X., Han, Y., Shi, W., Sun, S., et al. (2021). Fine Polystyrene Microplastics Render Immune Responses More Vulnerable to Two Veterinary Antibiotics in a Bivalve Species. Mar. Pollut. Bull. 164, 111995. doi:10.1016/ j.marpolbul.2021.111995

Zhu, T., Zhou, M., Yang, S., Wang, Z., Wang, R., Wang, W., et al. (2018). Occurrence and Ecological Risk of Sulfonamide Antibiotics in the Surface
Water of the Weihe Xi'an Section. Yellow River 40, 85-91. doi:10.3969/ j.issn.1000-1379.2018.12.020

Zhu, X., Wang, X., Wang, L., Fan, X., Li, X., and Jiang, Y. (2020). Biodegradation of Lincomycin in Wastewater by Two-Level Bio-Treatment Using Chloroperoxidase and Activated Sludge: Degradation Route and EcoToxicity Evaluation. Environ. Technology Innovation 20, 101114. doi:10.1016/j.eti.2020.101114

Zimmermann, P., and Curtis, N. (2020). Effect of Intrapartum Antibiotics on the Intestinal Microbiota of Infants: a Systematic Review. Arch. Dis. Child. Fetal Neonatal. Ed. 105, 201-208. doi:10.1136/archdischild-2018-316659

Conflict of Interest: The authors declare that the research was conducted in the absence of any commercial or financial relationships that could be construed as a potential conflict of interest.

Copyright (c) 2021 Liu, Tan, Zhang, Tian and Ma. This is an open-access article distributed under the terms of the Creative Commons Attribution License (CC BY). The use, distribution or reproduction in other forums is permitted, provided the original author(s) and the copyright owner(s) are credited and that the original publication in this journal is cited, in accordance with accepted academic practice. No use, distribution or reproduction is permitted which does not comply with these terms. 